\title{
Verifying a signature architecture: a comparative case study
}

\author{
David Basin ${ }^{1}$, Hironobu Kuruma ${ }^{2}$, Kunihiko Miyazaki², Kazuo Takaragi ${ }^{2}$, Burkhart Wolff ${ }^{3}$ \\ ${ }^{1}$ ETH Zürich, IFW C 49.1, 8092 Zürich, Switzerland \\ ${ }^{2}$ Hitachi, Ltd., Systems Development Laboratory, Kawasaki, Japan \\ ${ }^{3}$ ETH Zürich, IFW C 46.1, 8092 Zürich, Switzerland
}

\begin{abstract}
We report on a case study in applying different formal methods to model and verify an architecture for administrating digital signatures. The architecture comprises several concurrently executing systems that authenticate users and generate and store digital signatures by passing security relevant data through a tightly controlled interface. The architecture is interesting from a formal-methods perspective as it involves complex operations on data as well as process coordination and hence is a candidate for both data-oriented and process-oriented formal methods.

We have built and verified two models of the signature architecture using two representative formal methods. In the first, we specify a data model of the architecture in $Z$ that we extend to a trace model and interactively verify by theorem proving. In the second, we model the architecture as a system of communicating processes that we verify by finite-state model checking. We provide a detailed comparison of these two different approaches to formalization (infinite state with rich data types versus finite state) and verification (theorem proving versus model checking). Contrary to common belief, our case study suggests that $Z$ is well suited for temporal reasoning about process models with complex operations on data. Moreover, our comparison highlights the advantages of proving theorems about such models and provides evidence that, in the hands of an experienced user, theorem proving may be neither substantially more time-consuming nor more complex than model checking.
\end{abstract}

Keywords: Formal methods; Comparison; Theorem proving; Model checking; Security; Case study

\section{Introduction}

While there is increasing consensus about the usefulness of formal methods for developing and verifying critical systems, there are many options and schools of thought on how best to do this. Formal methods can be loosely characterized along different dimensions in terms of what views of a system they emphasize, the proof techniques used, etc. When most of the system's complexity stems from the way that processes interact, and the data manipulations are comparatively simple, then the use of a process-oriented modeling language, like a process algebra or some kind of communicating automata, is typically favored and model checking is the preferred means of verification. On the other hand, when system data is structured into rich data types (e.g., formalizing problem domains, interface requirements, and the like) that are subject to complex manipulations, then data-oriented modeling languages are considered superior and verification is carried out by theorem proving. But what about systems whose design encompasses both complex data and nontrivial interaction and whose requirements speak about both the operations on data and their temporal ordering? Here there is less consensus and the options 
available include using abstraction to simplify the data model to enable model checking, theorem proving, and even combining formal methods.

In this paper, we look at an example of one such system: a security architecture used for a digital signature application. The architecture is based on the secure operating system DARMA (Hitachi's platform for Dependable Autonomous hard Realtime MAnagement) [ASS ${ }^{+}$99], which is used to control the interaction between different subsystems, running on different operating platforms. In particular, DARMA is used to ensure data integrity by separating user API functions, which run on a potentially open system (e.g., connected to the Internet), from those that actually manipulate signature-relevant data, which run on a separate, protected system. Any model of this architecture must formalize both the processes that run on the different platforms and the data that they manipulate to produce signatures. Moreover, the modeling formalism must be capable of formalizing data-integrity requirements, expressed as temporal properties about how the different data stores should change.

We present two models of the signature architecture: one based on a data model formalized using Z [Spi92], and the other as a system of communicating processes formalized in PROMELA, the input language to the Spin model checker [Hol04]. In both languages, we are faced with the question of how to model the two aspects-rich data combined with process interaction-and verify the resulting models. In the $\mathrm{Z}$ model, we formalize a classical data model that describes the architecture in terms of component states and state transitions. Afterwards, we exploit the fact that $Z$ is a very rich specification language, and we extend the data model to a simple process model that describes the system's semantics in terms of the set of its traces. This provides a basis for naturally formalizing the system's security requirements as trace requirements. The main challenge then is verification, which requires interactively establishing invariants by induction over the set of system traces. In contrast, in our PROMELA model we focus on processes and their interactions. To verify the resulting model automatically, we simplify the data model such that the resulting system is finite state. The main challenges here are making this simplification, formalizing the properties that the resulting model should fulfill, and managing the complexity of model checking. Our case study provides concrete examples of these problems and how we have handled them.

Contributions Our main contribution is to provide a detailed comparison of these two different approaches to formalization (infinite state with rich data types versus finite state and process-oriented) and verification (theorem proving versus model checking). Our account is both quantitative and qualitative and sheds light on the relative strengths and weaknesses of the two approaches. Perhaps surprisingly, our experience in this case study is that, in the hands of an experienced user, theorem proving is neither substantially more time-consuming nor more complex, and in some regards it is considerably simpler, than working with a process-oriented view alone using a model checker. Moreover, we document a number of tradeoffs where the additional complexity is counterbalanced by additional benefits, for example, a more general architecture, stronger theorems, and an increased confidence in the system gained by formalizing and proving system invariants.

Our second contribution concerns the suitability of $Z$ for formalizing process-oriented models and requirements. We show here how the use of a sufficiently expressive data-modeling language provides a foundation for formalizing a trace-based model of process interaction. The ideas here are general and should carry over to other ways of composing processes. It follows that there is no need to resort to different formal methods to formalize and combine the different system views since this can all be done within $\mathrm{Z}$ itself. The practical benefit of this is not only the simplicity of a single formal method, but also the direct use of general-purpose tools. An example of this is the HOL-Z environment for the Isabelle theorem prover, whose use we describe in this paper.

Organization In Sect. 2, we provide an informal overview of both the signature architecture and its security requirements. In Sects. 3 and 4, we describe how we used Isabelle/HOL-Z and Spin, respectively, to model and verify the architecture. In Sect. 5, we compare approaches and in Sect. 6 we discuss related work and draw conclusions.

Note that in the interest of brevity, only illustrative aspects of the models and proofs are presented. All definitions and complete proof scripts for the two case studies are given in [BKTW04] and [Pro05].

\section{The signature architecture}

\subsection{Overview}

The signature architecture is based on two ideas. The first is that of a hysteresis signature [SM02], which is a cryptographic approach designed to overcome the problem that, for some applications, digital signatures should 


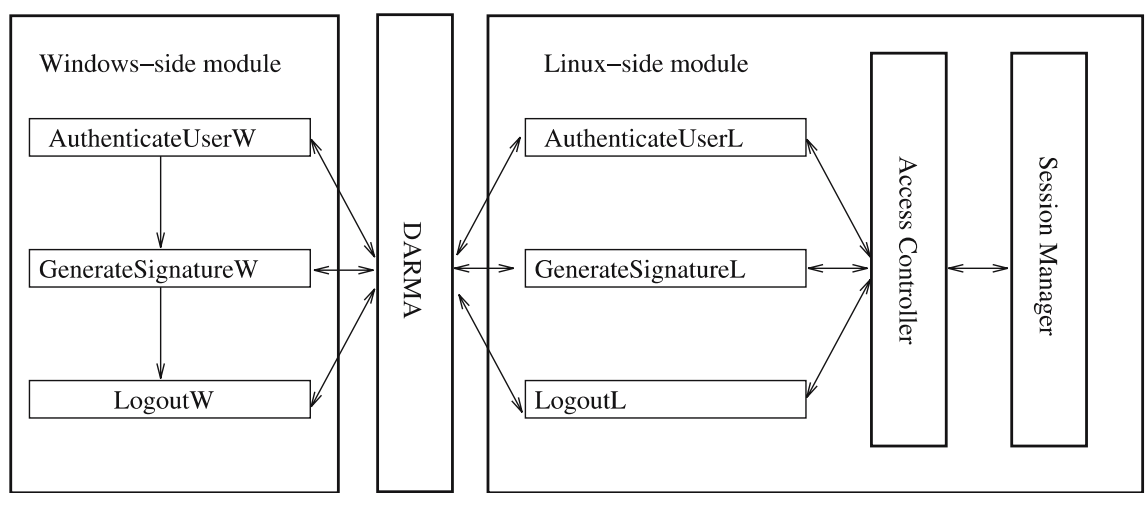

Fig. 1. The signature architecture

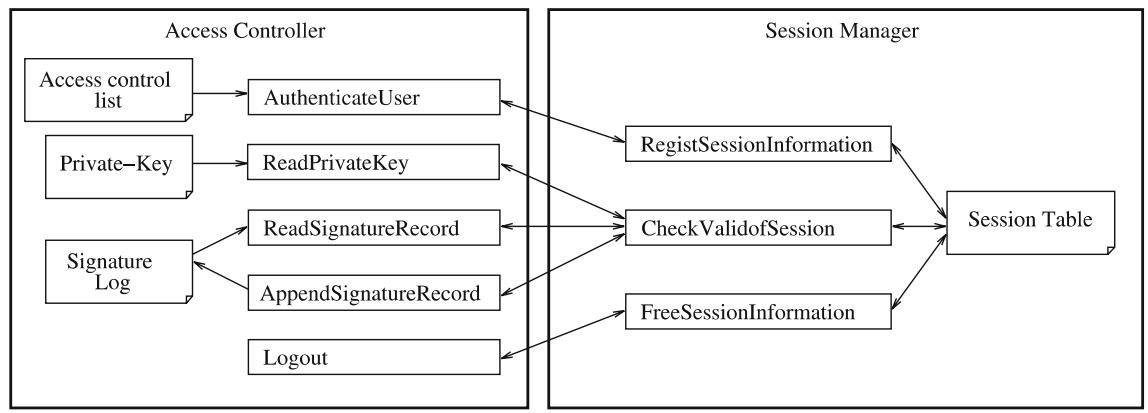

Fig. 2. The access controller and session manger modules

be valid for very long time periods. Hysteresis signatures address this problem by chaining signatures together so that the signature for each document signed depends on hash values computed from all previously signed documents. These chained signatures constitute a signature log and to forge even one signature in the $\log$ an attacker must forge (breaking the cryptographic functions behind) a chain of signatures.

The signature system reads the private keys of users from key stores, and reads and updates signature logs. Hence, the system's security relies on the confidentiality and integrity of this data. The second idea is to protect these using a secure operating platform. For this purpose, Hitachi's DARMA system [ASS ${ }^{+}$99] is used to separate the user's operating system (in practice, Windows) from a second operating system used to manage system data (e.g., Linux). This compartmentalization plays a role analogous to network firewalls, but here the two systems are protected by controlling how functions in one system can call functions in the other. In this way, one can precisely limit how users access the functions and data for hysteresis signatures that reside in the Linux operating system space.

Our model is based on a 13 page Hitachi document, which describes the signature architecture using diagrams (like Figs. 1 and 2) and text, as well as discussions with Hitachi engineers.

\subsection{Functional units and dataflow}

The signature architecture is organized into five modules, whose high-level structure is depicted in Fig. 1. The thick-lined boxes represent modules and the thin-lined boxes represent individual functions.

The first module contains three functions, which execute in the operating system space of the user. We call this the "Windows-side module" to reflect the (likely) scenario that they are part of an API available to programs running under the Windows operating system. These functions are essentially proxies. When called, they forward their parameters over the DARMA module to the corresponding functions in the second, protected system, which is here called the "Linux-side module", again reflecting a likely implementation. There are two additional (sub)modules, each also executing on the second system, which package data and functions for managing access control and sessions. 


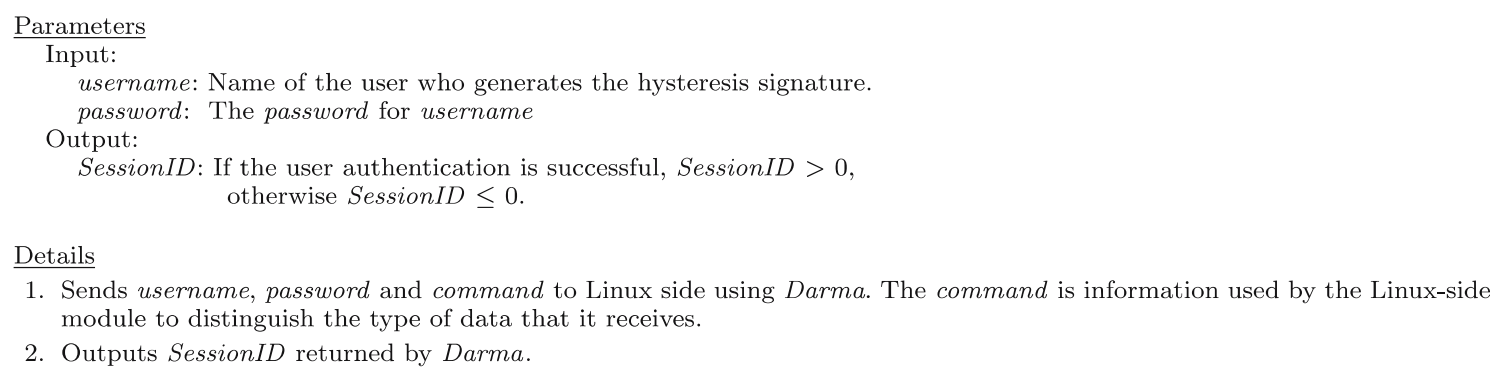

Fig. 3. Interface description for AuthenticateUser W

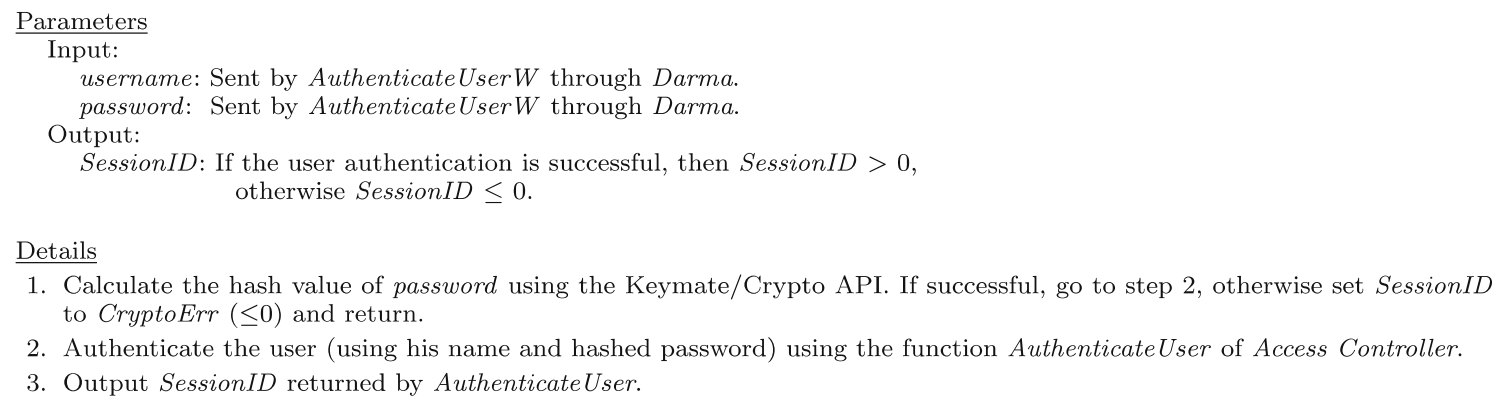

Fig. 4. Interface description for AuthenticateUserL

To create a hysteresis signature, a user takes the following steps on the Windows side:

1. The user application calls AuthenticateUser $W$ to authenticate the user and generate a session identifier.

2. The application calls GenerateSignature $W$ to generate a hysteresis signature.

3. The application calls Logout $W$ to logout, ending the session.

As explained above, each of these functions uses DARMA to call the corresponding function on the Linux side and DARMA serves to restrict access from the Windows side to only these three functions. The Linux functions themselves may call any other Linux functions, including those of the Access Controller, which controls access to data (private keys, signature logs, and access control lists). The Access Controller in turn uses functions provided by the Session Manager, which manages session information (SessionID, etc.), as depicted in Fig. 2.

The Hitachi documentation provides an interface description for each of these functions. Two representative examples are presented in Figs. 3 and 4. These are the descriptions of the functions AuthenticateUser $W$ and AuthenticateUserL. The former calls DARMA and returns a session identifier while the latter does the actual work of checking the password and communicating with the access controller.

\subsection{Properties}

The Hitachi documentation also states three requirements that the signature architecture should fulfill. These state that authenticated users are limited to generating one signature (with their private key) per authentication.

R1. The signature architecture must authenticate a user before the user generates a hysteresis signature.

R2. The signature architecture shall generate a hysteresis signature using the private key of an authenticated user.

R3. The signature architecture must generate only one hysteresis signature per authentication.

We will subsequently see how to model these requirements as properties of traces in both $\mathrm{Z}$ and linear temporal logic. 


\section{Modeling and Verification with Isabelle/HOL-Z}

\subsection{HOL-Z}

For our first model, we used $\mathrm{Z}$ as our modeling language and the HOL-Z environment for theorem proving. As $Z$ is well established and extensively documented, e.g., [Int, Spi92, WD96], we will assume that the reader has basic familiarity with it. HOL-Z [BRW03] is an environment built upon the Isabelle/HOL system [NPW02]. The HOL-Z environment provides a front end for creating "literate specifications", where specifications are mixed with informal explanations and are constructed as $\mathrm{IT}_{\mathrm{E}} \mathrm{X}$ documents, typeset using standard $\mathrm{Z}$ macros and idioms. These specifications are processed by HOL-Z and translated into a conservative shallow embedding of $Z$ in HOL. HOL-Z also provides tactic support tailored to reasoning about $Z$ specifications and implements various verification and refinement techniques.

\subsection{The data model}

Our formalization of the signature architecture's state and operations is standard and closely follows Hitachi's informal specification. We formalize a state schema for each of the different system modules and an operation schema for each function, based on their informal description.

State schemas. As examples, we present two state schemas: the session manager and DARMA. The session manager maintains a session table (session_table) and the set of active session identifiers (session_IDs), i.e. those session identifiers currently in use. A session tables associates user names and session identifiers with information on access permissions for keys and the signature log. The access permissions prevent, for example, two signatures from being generated within the same session.

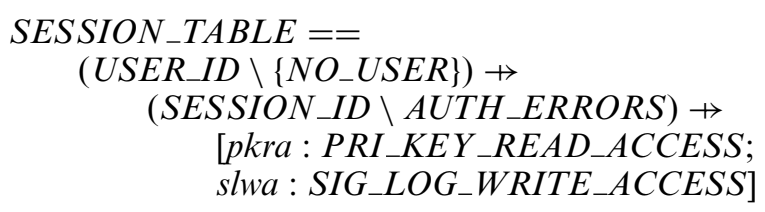

In this definition, USER_ID, SESSION_ID, PRI_KEY_READ_ACCESS, and SIG_LOG_WRITE_ACCESS are the types of user identifiers, session identifiers, and the permissions to access the private keys and the signature log, respectively. NO_USER and AUTH_ERRORS are constants representing error elements. The state of the session manager is formalized by the following $\mathrm{Z}$ schema:

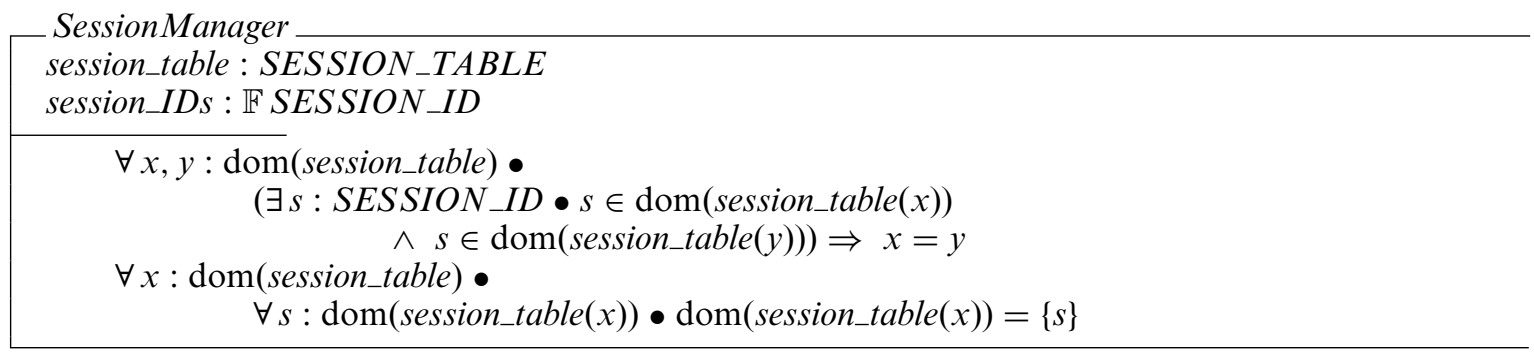

In general, a $\mathrm{Z}$ schema has a declarative part (above the line) and a predicate part (below the line). The declarative part specifies the schema's signature as a collection of typed fields, as in a record. The semantics of a $Z$ schema is the set of those records that fulfill the predicate part. In the above schema, the predicate part states that a session identifier is associated with at most one user identifier and, conversely, that each user identifier is associated with at most one session identifier. It follows that each authenticated user has exactly one unique session identifier. The authenticated users are therefore characterized by the "active sessions", i.e. by dom(session_table).

The DARMA module serves as a communication medium. It can be understood as a record containing shared variables into which both clients and the server "read" and "write" according to their needs. These shared variables record which of the three Windows-side functions are called along with its arguments and the return value from the Linux side. Part of this schema is given below, where we have elided declarations for the arguments and return values for the signature generation and logout functions. 


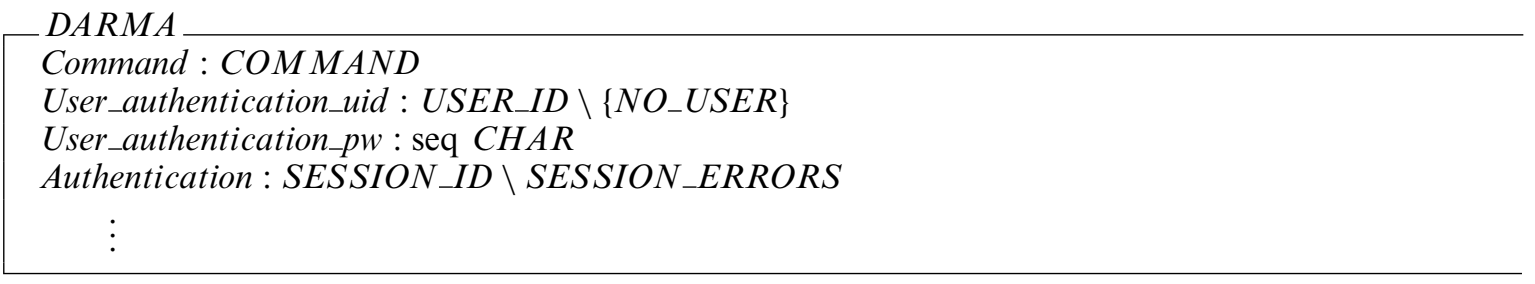

Operation schemas. Each of the module functions is associated with an operation schema. The association is mostly straightforward. However, one aspect that does require explanation is how we model the input and output to these functions. To do this, we explicitly identify the schema's local input and output variables (respectively postfixed by "?" and "?", following the standard Z convention) with their DARMA counterparts and use equality to mimic an assignment. ${ }^{1}$ We illustrate this below, for the module functions AuthenticateUser $W$ and AuthenticateUserL, which were described in Sect. 2.2.

The schema AuthenticateUser $W$ models the identically named function, given in Fig. 3. This function is quite simple and essentially acts as a proxy, forwarding values over DARMA. Hence the only thing to model is this communication.

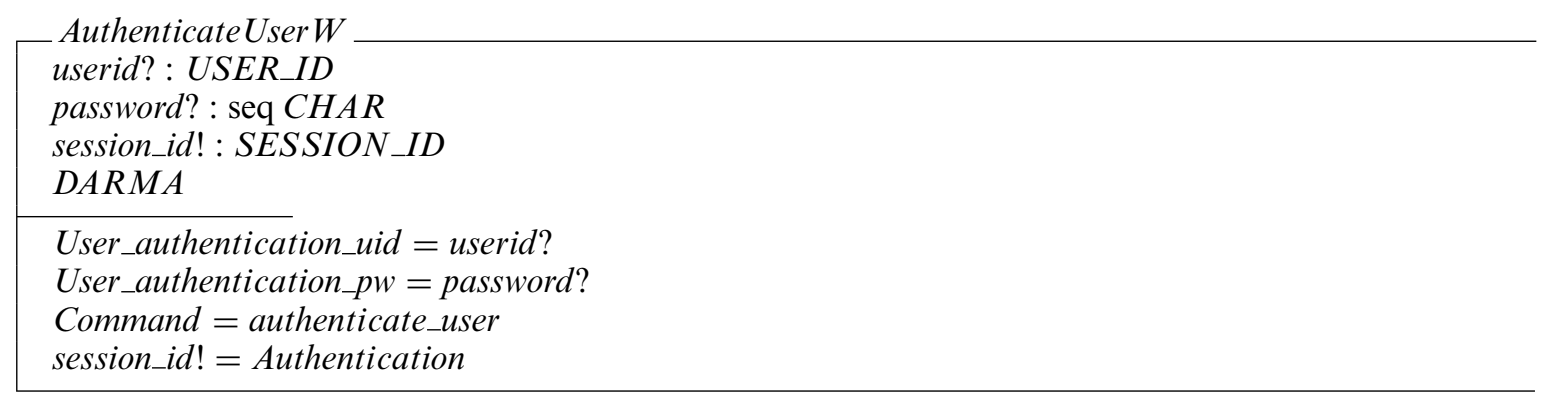

Here the variables User_authenticate_uid, User_authenticate_pw, Command, and Authentication are state variables from the DARMA state schema, imported in the declarative part. The first two are assigned to the input values userid? and password?, modeling user input. Command represents the name of the function called, named here by the constant authenticate_user. Finally the output of the schema, session_id!, is assigned Authentication, representing communication from DARMA (as we will see below, this represents the output of AuthenticateUserL).

The actual work in authenticating users and registering session information is carried out on the Linux side by AuthenticateUserL. Our operation schema here formalizes the description given in Fig. 4. The operation depends on the states of the components SessionManager, HysteresisSignature, and AccessController. This is formalized by importing the corresponding schemas into the declarative part of the schema using Z's $\Delta$ and $\Xi$ operators: $\Delta$ expresses that the operation may change the component's state, whereas $\Xi$ specifies that the state does not change. The predicate part directly reflects the informal description: Step 1 is formalized by the test if the generation of a hash value is successful. Step 2 is modeled in the first else branch, which calls the auxiliary function AuthenticateUser (e.g., with the hash value of the user's password) and which returns either a new session identifier or an error value. The remainder of the specification formalizes how to proceed, depending on whether the hash calculation and authentication succeeded or failed. In the former case (Authentication $\notin$ AUTH_ERRORS), the session manager's state is updated: the session table records, for this user identifier and session identifier, the right to read the user's private key and to update the signature log, and the set of session identifiers is updated with the new session identifier. In the latter case (Authentication $\in$ AUTH_ERRORS), the session manager's state is unchanged. Note that the result of AuthenticateUser $L$ is stored both in the output SessionID! and in the DARMA variable Authentication.

\footnotetext{
1 Logically, the input and output variables are determined by the DARMA state and could be eliminated. However, not only do they clarify the information flow, they also help to maintain the correspondence between our formal specification and Hitachi's informal interface descriptions (see Figs. 3 and 4) with their explicit inputs and outputs. Note too that, as is standard for Z, reference to input and output, as well as other imperative notions like assignment, is just a conceptual convenience; the semantics of $\mathrm{Z}$ schemas is, of course, the standard declarative one, given by sets of records.
} 


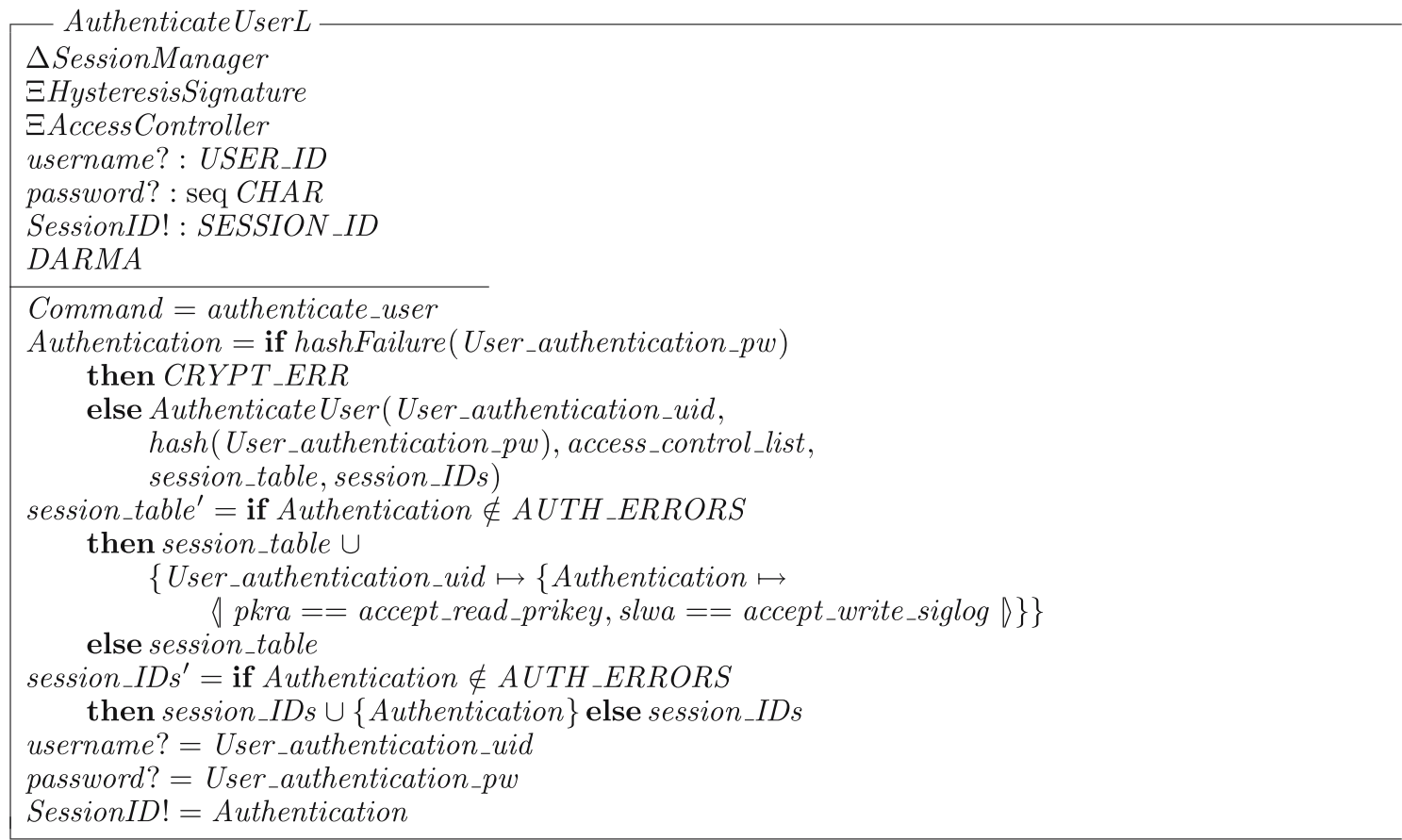

The auxiliary functions used in the above schema are defined using Z's axiomatic definitions. For example, hash is simply specified as a uninterpreted function over character sequences.

$$
\text { hash : seq CHAR } \rightarrow \text { seq CHAR }
$$

For the proofs that the signature architecture satisfies its requirements, no further properties of hash are needed. Note that this formalization models what is often called the "perfect cryptography assumption" in the security protocol community. This assumption says, for example, that you can only decrypt an encrypted message if you possess the appropriate decryption key, and you can only analyze the contents of a hashed message hash $(A)$ if you possess $A$ itself. This is the case here as there is no function that can be applied to $h a s h(A)$ to compute $A$, i.e., hash is one-way. Similarly, hashFailure is axiomatized as an uninterpreted predicate. ${ }^{2}$

\section{hashFailure $: \mathbb{P}($ seq $C H A R)$}

In contrast, our definition of AuthenticateUser specifies its concrete behavior. This function checks the user identifier and the hashed password against an access control list. In the case of a successful authentication, the function RegistSessionInformation is used to generate a fresh session identifier; otherwise the return value is an error element from $A U T H \_E R R O R S$.

\footnotetext{
2 The Hitachi specification is based on a particular cryptographic API that allows hash functions to fail, e.g., if the pointer to the string to be hashed is null. See also Figure 4.
} 


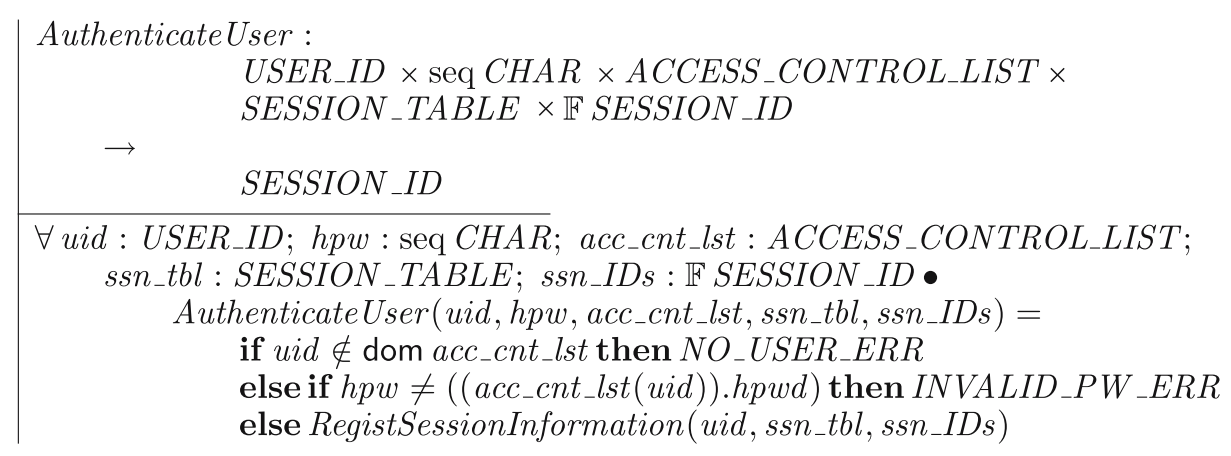

We will refrain from giving further details at this point, as the above should suffice to illustrate the main modeling ideas.

\subsection{The process model}

In general, there are many possible ways of enriching a data model with process-oriented aspects, ranging from the use of combined (data/process-oriented) formal methods, e.g., [Fis97, SD97], to working with a fixed notion of abstract machine and execution semantics, e.g., [Abr96]. In our case, we proceed by formalizing the system traces within $\mathrm{Z}$.

Architecture as transition system. We use Z's schema calculus to "wire together" the parts of our data model into an architectural description by specifying how the Windows-side operations interact with the Linux-side operations over DARMA. First, we separately collect all the client-side and server-side operations. We use schema disjunction here to model nondeterministic choice. The two resulting transition relations, ClientOperation and ServerOperation, model a system where the Windows-side and Linux-side functions may be called in any order and with any values, valid or invalid. Afterwards, we use schema conjunction to model the parallel composition of the client-side operations with the server-side operations and we use existential quantification, again in Z's schema calculus, to hide the shared DARMA state. ${ }^{3}$ This models synchronous internal communication between the sides. (Internal communication within each side is not modeled here.) The resulting architectural description defines a global transition relation.

$$
\begin{aligned}
& \text { ClientOperation }==\text { AuthenticateUser } W \vee \text { GenerateSignature } W \vee \text { Logout } W \\
& \text { ServerOperation }==\text { AuthenticateUserL } \vee \text { GenerateSignatureL } \vee \text { LogoutL } \vee \text { NopOperationL }
\end{aligned}
$$$$
\text { System }==\exists D A R M A \bullet \text { ClientOperation } \wedge \text { ServerOperation }
$$

Note that NopOperationL models a "no-op" operation on the Linux side by simply stuttering the Linux-side state. It results when DARMA is called from the client side, but a client-side error occurs and the step is aborted. Note that the transition relation System allows for two or more signature generation steps for the same session. However, due to flags in the session table, an attempted second signature is specified to produce an internal error, which results in a stuttering step (cf. Sect. 3.2).

Afterwards, we specify the global state of the system by composing the states of the system components using schema conjunction. (Here HysteresisSignature formalizes the part of the Linux-side module's state that manages the signature logs, while the AccessController maintains a table with the private keys of users.) Similarly, we specify the initial state, given schemas (not shown here) specifying the initial states of the different modules.

GlobalState $==$ SessionManager $\wedge$ HysteresisSignature $\wedge$ AccessController

Init $==$ SessionManagerInit $\wedge$ HysteresisSignatureInit $\wedge$ Access ControllerInit

\footnotetext{
${ }^{3}$ Schema operations such as conjunction and disjunction combine the underlying signatures of their operands. In contrast, existential quantification in Z's schema calculus hides the signature of the quantified schema.
} 
System traces. The schema System formalizes a transition relation, whose state variables range over the input/output variables of all operation schemas (e.g., variables like username? and SessionID! from AuthenticateUser $W$ ). To reason about the system behavior, what we actually need is a transition relation expressed in terms of just those variables in GlobalState (e.g., state variables such as session_table and session_IDs from the state schema SessionManager). Hence, to proceed, we project the transition relation System to those state variables in GlobalState by existentially quantifying over the remaining variables, like input and output variables. This construction can be elegantly formalized using Z's schema comprehension:

$$
\text { Next }==\{\text { System } \bullet(\theta \text { Global State, } \theta \text { GlobalState })\} .
$$

This builds the relation that consists of pairs ( $\theta$ GlobalState, $\theta$ GlobalState' $)$, whose components formalize the variable tuples (so-called characteristic bindings in Z) in the pre-state and post-state.

The pair (Init, Next), together with the collection of global states, constitutes a Kripke structure and induces a set of traces (or runs) in a canonical way. A trace is represented by a function that describes how the global state of the system can evolve over time.

$$
\text { Traces }==\{f: \mathbb{N} \rightarrow \text { GlobalState } \mid f(0) \in \text { Init } \wedge(\forall i: \mathbb{N} \bullet(f(i), f(i+1)) \in N e x t)\}
$$

As is standard, in our formalization, a trace represents an infinite sequence of states. Note that any finite trace can be extended to an infinite one simply by stuttering the last state. This is possible in our setting as some operations do not change GlobalState in erroneous cases (for example, login for non-existing users).

\subsection{Formalizing the security requirements}

The architecture's informal requirements, given in Sect. 2.3, are phrased in terms of temporal relationships between system events. For example, (R1) states that "the signature architecture must authenticate a user before the user generates a hysteresis signature." This, and the other two requirements, can be formalized as a set of traces that constitutes a safety property over a set of events and we can formalize the correctness of the architecture by stating that each such property holds for every system trace.

To proceed this way, we must first formalize the relevant events. In model checking, it is common to associate events with different states in a transition system, which correspond to execution events like calls to particular functions. Unfortunately, this leaves open the question of where these events are actually generated. Moreover, it is not well suited to a more abstract, declarative approach to modeling where, rather than program points, there are only sequences of program states. Here we will take an alternate, less operational approach. We introduce abstract event predicates that characterize the state changes associated with events, i.e., they specify the effect of events rather than their cause. An event predicate, therefore, is a (possibly parameterized) relation over pairs of states that characterizes when a relevant state change occurs.

Let us now turn to (R1), our first requirement. (R1) can be formalized in terms of three event predicates: the session table changes due to a user authenticating himself by logging in; the session table changes due to a user logging out; and the signature log changes due to the generation of a hysteresis signature for some user. Below is our axiomatic definition of these predicates.

$$
\begin{aligned}
& \text { In, Out, Sign : USER_ID } \rightarrow \text { (GlobalState } \leftrightarrow \text { GlobalState }) \\
& \forall \text { uid : USER_ID } ; s 1, s 2 \text { : GlobalState • } \\
& (s 1, s 2) \in \operatorname{In}(\text { uid }) \\
& \Leftrightarrow \text { uid } \notin \operatorname{dom}\left(s 1 . s e s s i o n \_t a b l e\right) \wedge \text { uid } \in \operatorname{dom}(\text { s2.session_table }) \wedge \\
& (s 1, s 2) \in \text { Out(uid) } \\
& \Leftrightarrow(\text { uid } \in \operatorname{dom}(s 1 \text {.session_table }) \wedge \text { uid } \notin \operatorname{dom}(\text { s2.session_table })) \wedge \\
& (s 1, s 2) \in \operatorname{Sign}(\text { uid }) \\
& \Leftrightarrow((\text { uid } \in \operatorname{dom}(s 1 \text {.signature_log }) \wedge \text { uid } \in \operatorname{dom}(s 2 \text {.signature_log }) \\
& \wedge(\text { s1.signature_log }(\text { uid }) \neq s 2 \text {.signature_log (uid }))) \\
& \vee((\text { uid } \notin \operatorname{dom}(s 1 \text {.signature_log }) \wedge \text { uid } \in \operatorname{dom}(\text { s2.signature_log }))))
\end{aligned}
$$

We can now directly formalize (R1) in terms of the relative positions (reflecting the relative time) where these predicates hold in the system traces. Our requirement states that at every point where a user changes the signature log, there exists a previous time point where he has logged in, and moreover he has not logged out since then. In other words, there must be a login for the user before the associated signature log entry is changed and his session 


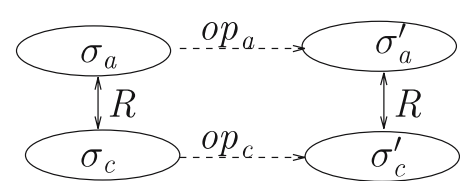

Fig. 5. General refinement diagram

must still be valid.

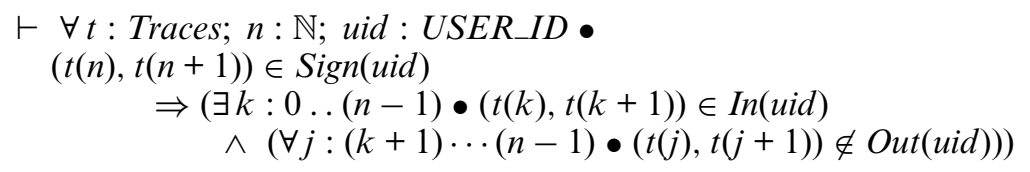

The other two requirements are formalized similarly.

We conclude with a remark on the close relationship between our use of the event predicates and refinement. These predicates can be understood as operation schemas on a more abstract system description level, which are refined by the concrete operations for logging in, logging out, and signing. For example, In can alternatively be formulated by the following operation schema:

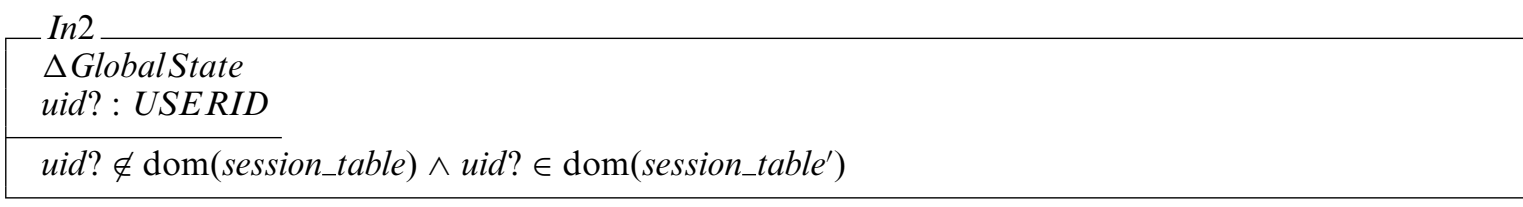

These abstract operation schemas can be related to the concrete operation schemas by a refinement relation. Roughly speaking, and as depicted in Fig. 5, refinement relates abstract operations $o p_{a}$ to concrete operations $o p_{c}$ by relating, under an abstraction relation $R$, the states of the abstract system $\sigma_{a}$ with the states of the concrete system $\sigma_{c}$. The technical details vary depending on which notion of refinement is chosen (e.g., forward and backward simulation [WD96]), and each imposes its own additional conditions, for example, that the domains and ranges of the operations are compatible via $R$, etc. In our setting, where event predicates are related to concrete operations, it is not difficult to check (and we have done so using Isabelle/HOL) that there is a simple refinement relation where $R$ is just the bijection on states and proving refinement amounts to showing that the abstract and concrete operations are related one-to-one. For example, the schema LogoutL implies Out and if two states in a system transition Next fulfill Out, then LogoutL is the only possible operation.

\subsection{Proofs}

All three requirements were proved using the proof environment for HOL-Z. In our comparison in Sect. 5, we provide statistics on our verification effort. Here we restrict ourselves to a few comments on its overall structure.

The verification required proving 173 theorems. Many of these were simple lemmas, for example, for simplifying expressions, which were then incorporated into Isabelle's automatic proof procedures. The bulk of the preparatory work centered around formalizing and proving (1) properties of operation schemas, (2) architecture decomposition theorems, and (3) global invariants.

With respect to (1), for each operation schema we stated and proved lemmas that characterize its preconditions, postconditions, and invariants in terms of its inputs, outputs, pre-state, and post-state. The theorems proved were of the form

$$
O P\left(\text { in }, \text { out }, \sigma, \sigma^{\prime}\right) \Rightarrow C O N D\left(\text { in }, \text { out }, \sigma, \sigma^{\prime}\right) \Rightarrow \Phi\left(\sigma, \sigma^{\prime}\right),
$$

where $O P$ is an operation schema, $C O N D$ a side-condition and $\Phi$ is one of:

$\operatorname{INV}\left(\sigma, \sigma^{\prime}\right)$, expressed in terms of (state variables from) the pre-state $\sigma$ and the post-state $\sigma^{\prime}$;

$\operatorname{PRE}(\sigma)$, expressing a condition on the pre-state $\sigma$; or

$\operatorname{POST}\left(\sigma^{\prime}\right)$, expressing a condition on the post-state $\sigma^{\prime}$. 
An example of such a lemma is the invariant

$\vdash$ AuthenticateUserL $\Rightarrow$ uid $: \operatorname{dom}($ session_table $) \Rightarrow$ session_table $($ uid $)=$ session_table(uid $),$

stating that when a user identifier is in the session table, its entries remain unchanged after another user is authenticated. Note that, as this example illustrates, HOL-Z is syntactically more liberal than Z. This invariant is a HOL-Z formula, but strictly speaking not a $\mathrm{Z}$ formula, since it combines $\mathrm{Z}$ schema expressions and predicate calculus expressions and it is not closed.

In general, the complexity of proving these lemmas ranged from easy (as in this case) to very high, both in terms of the conceptual work required to understand why they hold and in terms of the proof effort required in Isabelle.

With respect to (2), one of the main lemmas proved was an architecture decomposition theorem, which states that the signature architecture can make progress in exactly four ways:

1. an AuthenticateUser $W$ step occurs in parallel with an AuthenticateUserL step;

2. a GenerateSignature $W$ step starts and aborts due to an internal error while running in parallel with NopOperationL (a stuttering step on the Linux side);

3. a GenerateSignature $W$ step occurs in parallel with a GenerateSignature L step; or

4. a Logout $W$ step occurs in parallel with a Logout $L$ step.

By using the $\mathrm{Z}$ schema calculus, this theorem can be compactly expressed as:

$\vdash(\exists D A R M A \bullet$ AuthenticateUser $W \wedge$ AuthenticateUserL $) \vee$

$(\exists$ DARMA $\bullet$ GenerateSignature $W \wedge$ NopOperationL $) \vee$

$(\exists D A R M A \bullet$ GenerateSignature $W \wedge$ GenerateSignatureL $) \vee$

$(\exists$ DARMA $\bullet$ Logout $W \wedge$ LogoutL)

$\Leftrightarrow$ System.

This theorem explains in which ways synchronous communication over DARMA is possible. We use it in the right-to-left direction as a kind of "elimination rule" that uses case analysis to decomposes assumptions about steps in traces: if we have a trace $t$ and a system transition $(t(n), t(n+1))$, a property $P(t(n), t(n+1))$ holds if it holds for the four possible system transitions. Note that the complexity of the case split reflects the low complexity of the coarse-grained communication within the architecture: the client and server side operations synchronize in just four different ways. For a less tightly-coupled architecture, more cases would be needed, expressing all the ways that operations could occur.

With respect to (3), we proved a large number of global invariants, which are formulas of the form $\forall t$ : traces $\bullet$ $I N V(t(n), t(n+1))$. Examples of such invariants are that the signature log monotonically increases and that the domain of the session table and signature log are always bounded by the domain of the table of private keys. These lemmas, as well as the proofs of the three requirements, were proven by induction over the positions in a trace. In the inductive case, the architecture decomposition theorem was applied to decompose the step into possible cases. In each case, either other global invariants or relevant lemmas about properties of operation schemas were used to reason about the consecutive states. Hence, induction and decomposition served as the primary mechanism to reduce the reasoning about global invariants to standard reasoning about local preconditions, postconditions, and invariants of operations. In our experience, and perhaps in contrast to common belief, the proofs of these global invariants were not particularly difficult. The main proof effort was spent in establishing local invariants like $I N V\left(\sigma, \sigma^{\prime}\right)$, that is, carrying out conventional reasoning about preconditions and postconditions. We shall return to this observation and provide further perspective on it in Appendix B. An example of stating and proving a global invariant is given in Appendix C.

\section{Modeling and verification with spin}

The security properties of the signature architecture define authorized sequences of actions, i.e., properties of traces. This suggests building a process-oriented system model from the start that focuses on processes, relevant aspects of their internal computation, and their communication. We describe such a model in this section and verification by model checking. 


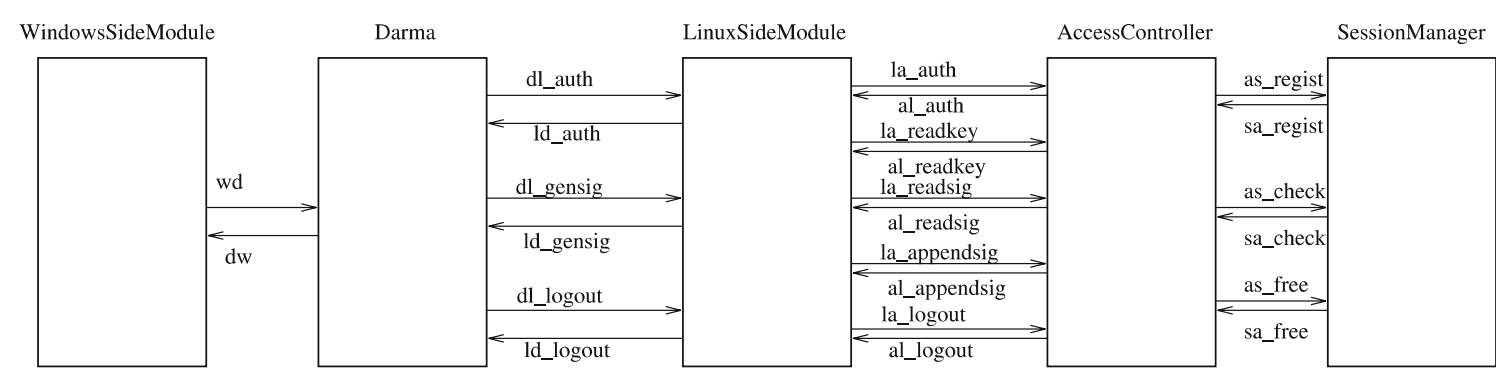

Fig. 6. Modules and Channels

\subsection{Spin}

There are a variety of formalisms and tools suitable for process modeling and verification. We have used Spin, one of the most advanced publicly available model checkers, to formalize and check our second model.

Spin is a model checker that supports the design and verification of distributed systems and algorithms. Spin's modeling language, called PROMELA (PROcess MEtaLAnguage), provides a C-like notation for formalizing processes, enriched with process-algebra-like primitives for expressing parallel composition and communication. Properties may be expressed in future-time LTL and Spin implements algorithms for LTL model checking. Appendix A provides a brief introduction to LTL; the PROMELA modeling constructs themselves will be introduced as needed, on-the-fly. For a detailed description of Spin, the reader should consult [Hol04].

\subsection{Abstraction}

As previously noted, one of the main challenges in building a process model is to formalize the system at the right level of abstraction. While this is true for modeling in general, it is particularly crucial when the objective is to arrive at a finite-state system whose properties can be verified by model checking. In the case of the signature architecture, the system's behavior depends on the data administered, e.g., the values of keys, session identifiers, hash values, and the like, which come from large or even infinite domains. To proceed, we must abstract these data domains into small finite sets, and model functions over data as functions over the corresponding finite sets.

The approach we take is to limit the environment in which the system can be used. In our Z model, the global transition relation System modeled interaction with an environment that could call any operation, in any order, and with any value. Whether the transitions represented actions associated with legitimate users, or attackers, was irrelevant, as was the number of such potential users. In our PROMELA model, we will restrict both the number of users that can interact with the signature architecture and the values with which they can call system functions.

In particular, we model the signature architecture as a system operating in an environment comprised of honest users and an attacker. The honest users use the system as intended while the attacker uses the system in perhaps unintended ways and, in particular, attempts to exploit and compromise the system. In both cases, the values with which these principals call system functions are restricted to finite domains. We build the overall system model from submodels that define processes for each of the different subsystems together with the processes that model the normal users and the attacker. We then prove that the desired security properties hold of the system, even in the presence of all the possible malicious actions that can be taken by the attacker. This is analogous to the approach taken in verifying security protocols [Pau98, $\left.\mathrm{RSG}^{+} 00, \mathrm{BMV} 05\right]$, where one explicitly models an active attacker who controls the computer network and proves that protocols achieve their properties despite the attacker's interference.

\subsection{Modeling communication}

Let's begin with communication. As suggested by Figs. 1 and 2, we can model the signature architecture in terms of five communicating modules. In the PROMELA model, we explicitly model communication; this is in contrast to our $\mathrm{Z}$ model (see Sect. 3.3), where communication is implicitly captured by the way that state transitions are forced to synchronize with DARMA on certain values in the definition of the global transition relation System. 
So here we model each module as a PROMELA process, where each process communicates with other processes over channels. A PROMELA channel is a buffer of some declared (finite) size that holds data of specified types. For each function in a module, we define two channels: one for modeling function calls and the other for modeling the return of computed values. This is depicted in Fig. 6, which names the channels used for passing data between processes. All channels are declared to have size zero, which models synchronous communication under PROMELA's semantics: the process sending data on a channel and the process receiving data from the channel must rendezvous, i.e., carry out their actions simultaneously.

As the figure shows, between Windows and DARMA we have just one calling channel $w d$ and one returning channel $d w .{ }^{4}$ This reflects that we have only one function in the Darma interface. This function is called by marshaling (i.e., packaging) the function arguments together, including the name of the function to be called on the Linux side. We model this by putting all these arguments on the channel. For example, the expression wd! Auth User, username, password (which occurs in our model of a normal user, given shortly), models that the function AuthenticateUser W calls Darma, instructing Darma to call AuthenticateUserL with the arguments username and password.

\subsection{Modeling system users}

We now explain our formalization of both normal system users and attackers. The description of the signature architecture in Sect. 2 describes how the system is intended to be used by normal users. As we will see, it is a simple matter to translate this description into a process that models such users.

The Hitachi documentation describes, in part, the powers and limitations of an attacker; in particular, an attacker cannot access functions on the Linux side. This is a starting point for our formalization of an attacker model, but it leaves many points open, for example, whether an attacker can operate within the Windows-side system as a legitimate user with a valid password, or if he is an outsider, without these abilities. Moreover, it is not specified what the attacker knows, can guess, or can feasibly compute.

One achieves the strongest security guarantees by proving the safety of a system in the face of the most general and powerful attacker possible. Hence, we model an attacker who cannot only function as a legitimate user of the system, but can also call functions in unintended ways, with arbitrary parameters. Moreover, he knows, or can guess or compute, the names of other users, messages, and message hashes, and of course he knows his own password. However, we assume he can neither guess the passwords nor the session identifiers of other users. If either of these were possible, then forging signatures would be trivial. An attacker who can guess the password of a user can authenticate himself to the system as that user and thereby generate signatures in the user's name. Moreover, the result of a successful authentication is a session identifier and an attacker who can guess these can hijack sessions by guessing identifiers currently in use.

We summarize these assumptions as follows:

1. The attacker can call AuthenticateUser W, GenerateSignature $W$, and Logout $W$ in any order.

2. The attacker is also a legitimate user with a user name and a password.

3. The attacker knows the names of all users and he can guess messages and message hashes.

4. The attacker can only give his (good) password or a bad guessed password.

5. The attacker cannot guess a good SessionID, i.e., one used by other users.

6. Generated SessionIDs are always good.

In our PROMELA model, we define sets of objects, namely finite intervals of natural numbers, for modeling the different kinds of objects in the problem domain: names, messages, hash values, and passwords. The key idea is to partition these sets into those things that are known by the attacker (or can be guessed or computed) and those that are not. For example, there is a set of user names, formalized by the set of natural numbers $\left\{M I N \_\right.$username, ..., MAX_username $\}$. We model that the attacker knows, or can guess, any of these names by allowing him to guess (by nondeterministically selecting) any number in this set. However, we partition the ranges corresponding to passwords and session identifiers so that the attacker can only guess "bad" ones, which are ones that are never assigned to normal users. In addition, the attacker also has a "good" password, which allows him

\footnotetext{
${ }_{4}$ Note that we ignore channels for calling the Windows functions since the functions that actually call AuthenticateUserW, GenerateSignature $W$, and Logout $W$ fall outside the scope of our model, that is, we do not consider either the calling context or how the results are used.
} 


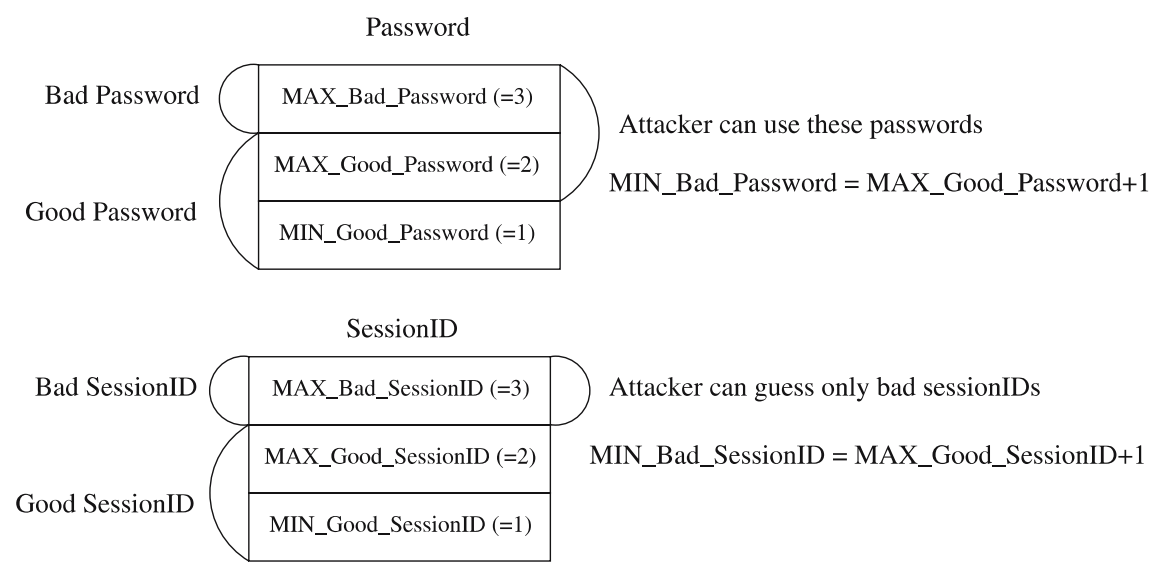

Fig. 7. Modeling passwords and session identifiers

to use the system as a normal user and generate a good session identifier. Figure 7 depicts this partitioning, with the concrete values that we later use when model checking. For example, the good passwords are $\{1,2\}$, where 2 represents the attacker's password. He can only guess passwords in the range $\{2,3\}$, where 3 models a bad password, i.e., one that does not belong to any normal user. As he cannot guess the password 1 , he cannot use the system (e.g., to generate a signature) as any user other than himself.

Given this abstraction, it is now a simple matter to model the actions of normal users and the attacker.

Normal users. Figure 8 shows our model ${ }^{5}$ of a normal user, which models the steps that such users take when using the signature architecture.

In lines 4 and 5 we model the different possible choices for system users and their messages. The macro setrandom ( $x$,lower,upper) is defined as:

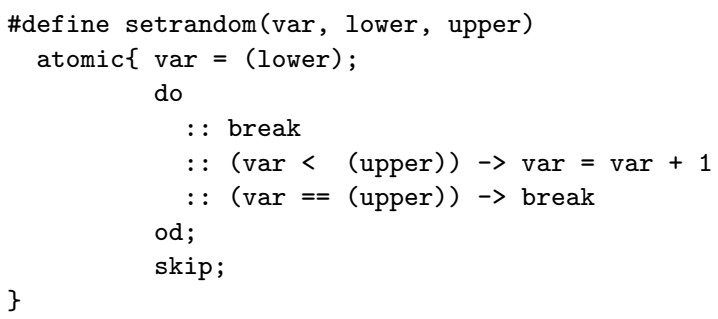

This routine executes as a single atomic step and uses nondeterministic choice within a loop to set $x$ to a value, lower $\leq x \leq$ upper. Hence lines 4 and 5 set the username and password to those of a normal user, chosen nondeterministically from the predefined ranges.

Afterwards, the user generates a hysteresis signature. The lines 8 and 9 model AuthenticateUser $W$, which models the equivalent of the identically named Z schema, which was presented in Sect. 3.2. Specifically, line 8 models the user calling Darma on the wd channel, specifying the execution of the Linux-side user authentication function, along with his username and password. Line 9 models the result returned on the $d w$ channel: a session identifier (whose value is greater than zero when authentication is successful).

On lines 11-12, a message from the space of possible messages is nondeterministically selected and its message hash is computed. We model Hash simply as the identity function. Although this does not satisfy the functional requirements of a cryptographic hash function, in particular, that it is a one-way function, it is adequate for establishing the stipulated properties of our process model, which only rely on passwords and session identifiers being unguessable. On line 14, the user calls Darma on the $w d$ channel, instructing Darma to generate a signature with the session identifier returned from the previous round of authentication and the message hash. The gener-

\footnotetext{
5 Model excerpts are taken verbatim from our PROMELA model, with the exception of pretty printing, line numbering, and minor simplifications for expository purposes.
} 


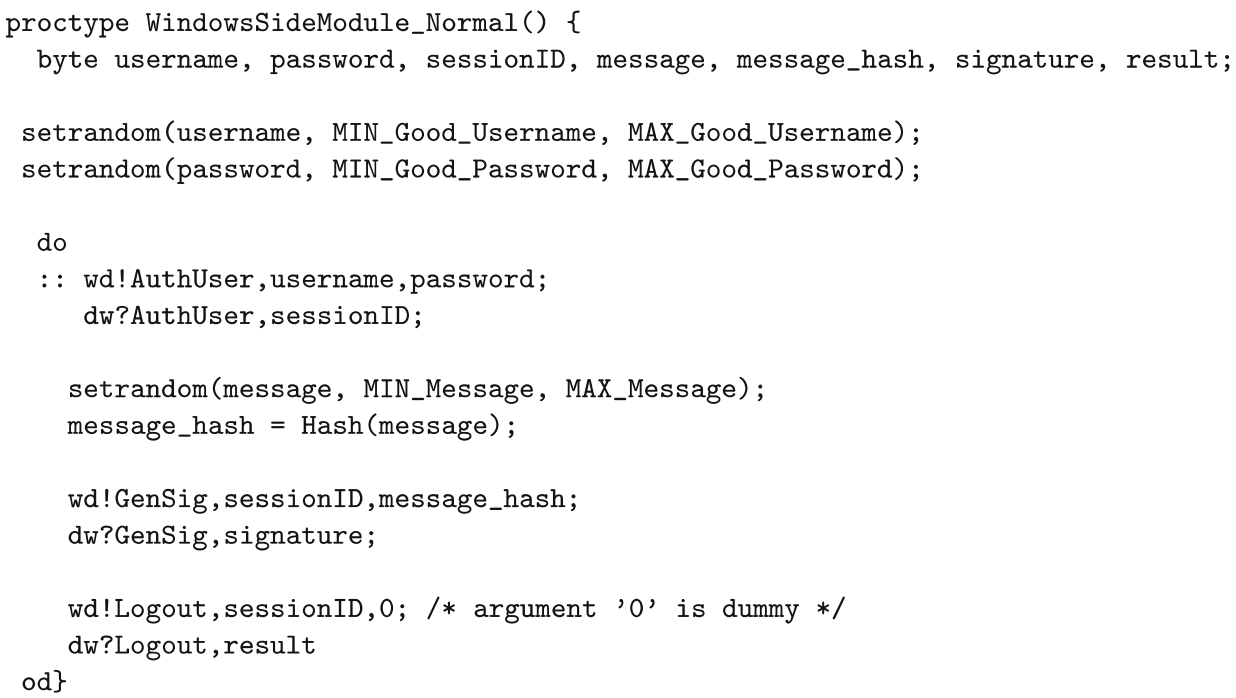

Fig. 8. User model

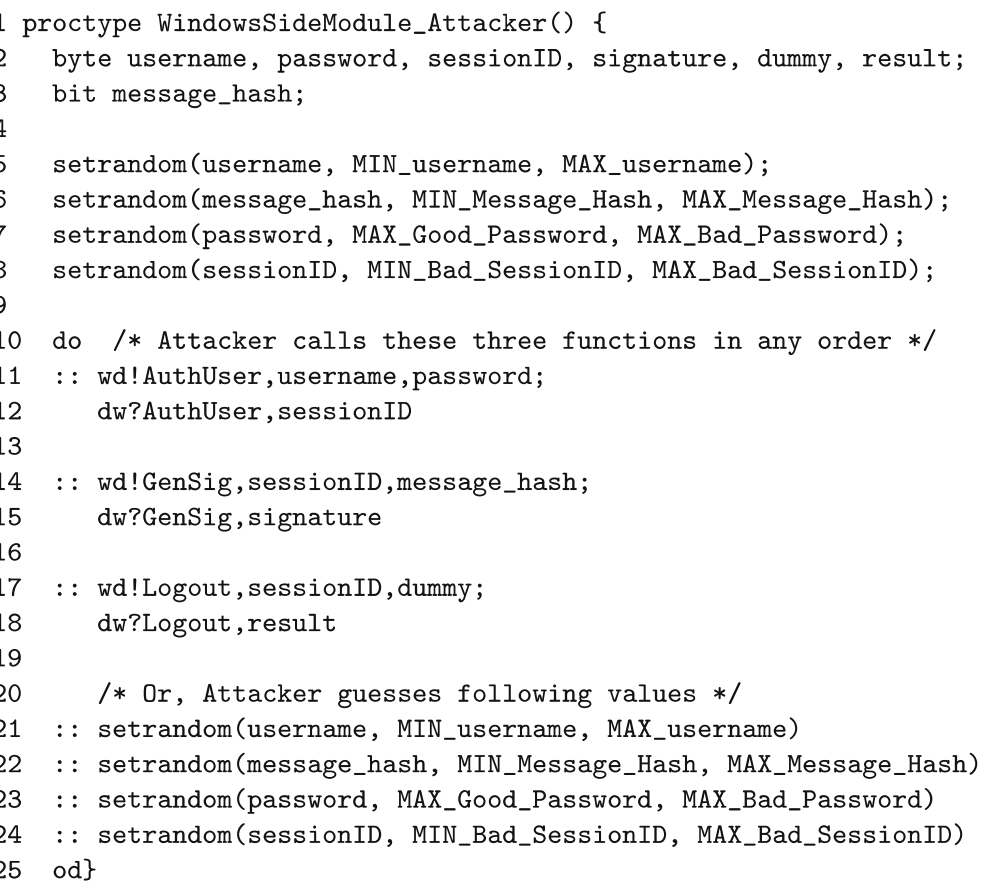

Fig. 9. Attacker model

ated signature is returned on line 15 . Note that the return value can also indicate an error, for example when the session identifier is invalid.

Lines 17-18 model the user logging out, which invalidates his session identifier.

The attacker. Figure 9 shows the PROMELA process that formalizes our attacker model. Here we see that the attacker can guess an arbitrary user name and message hash (lines 5-6). However, in accordance with the guessing model depicted in Fig. 7, he can only guess one good password (Max_Good_Password), which allows him to $\log$ in as a normal user, or bad passwords (line 7). Similarly, he can only guess bad session identifiers (line 8). 


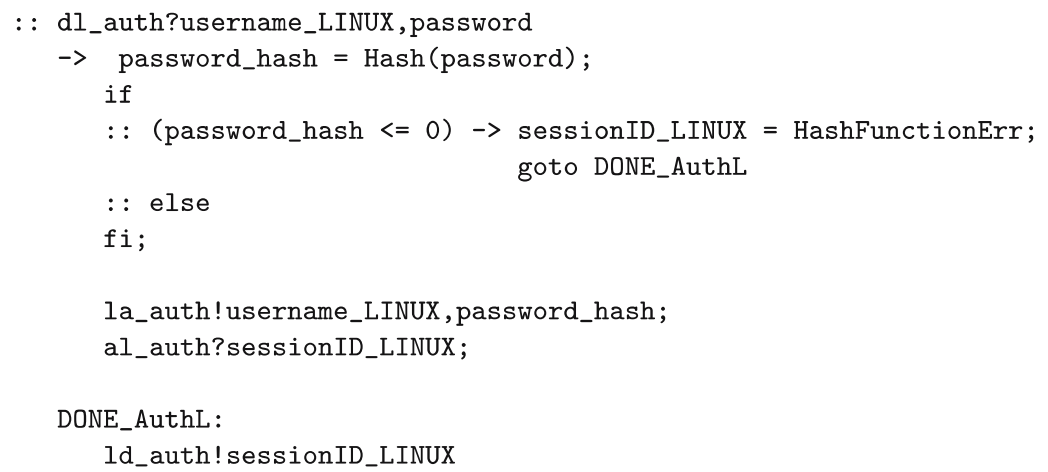

Fig. 10. AuthenticateUserL

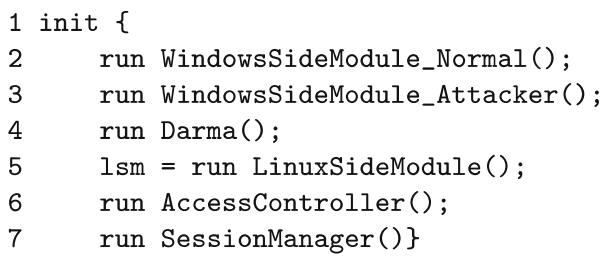

Fig. 11. Initialization process

Afterwards, we use a loop with nondeterministic choice to model the attacker repeatedly calling Darma (on the $w d$ channel) with these guessed values, in any order he likes. Alternatively, as formalized by the last four actions, he can guess new values at any point in time.

This example again illustrates the power of nondeterminism in a process-oriented modeling language. As with the user model, we use it to leave open which values are taken on by variables. This models a system where these variables can take on any value from the specified sets at system runtime. In addition, we use nondeterminism to describe the different possible actions that can be carried out by a user, while allowing the actions to be ordered in any way. This can be contrasted to our $\mathrm{Z}$ model which uses relations to formalize a nondeterministic transition relation. In the case of model checking, this nondeterminism typically leads to verification problems with large search spaces whereas in theorem proving it results in the need to perform case splits.

\subsection{Modeling module functions}

The majority of our PROMELA model describes the different functions contained in the system modules. As the Windows-side functions (e.g., AuthenticateUser $W$ ) have been straightforwardly modeled as calls to DARMA within the user processes, we are left with modeling the Linux-side functions. We model each of them in a standard process-oriented way, by making the control flow of each function explicit, as well as the operations on state variables, and the synchronization with other processes. Functions operating on state variables, which range over finite domains, approximate their counterparts operating over infinite domains, as previously described.

As a representative function, we return to AuthenticateUser L, first described in Sect. 2.2 and specified in Z in Sect. 3.2. Figure 10 shows the part of the PROMELA process that models this function (the module also contains definitions for the other Linux-side functions). This directly models the three steps explained in Sect. 2.2: calculate a hash value (lines 2-7), authenticate the user (lines 9-10), and return the session identifier (line 13).

\subsection{Putting It all together}

We build the overall model by composing in parallel the processes defined above. Namely, we compose the two processes formalizing the Windows-side module (as used by normal users and by the attacker) and the processes for the remaining modules. This is depicted in Fig. 11. Note that we associate an identifier $l s m$ with the process 
executing the Linux-side module. This will be used during verification to refer to particular labels in an invocation of the LinuxSideModule process, as described in the next section.

\subsection{Model checking}

We have used the Spin model checker to verify that our model satisfies the security properties listed in Sect. 2.3. Prior to describing this verification, we first recount how Spin model checking works in general.

Spin can be used to establish that a formula $\phi$ of future-time linear temporal logic holds of a model $\mathcal{M}$, i.e., $\mathcal{M} \models \phi$. The formula $\phi$ formalizes the desired, or "good", system behavior and $\mathcal{M}$ is a Büchi automaton derived from the PROMELA model. For model checking with Spin, one negates $\phi$, thereby expressing "bad" system behavior, which in our case is the set of traces that represents security violations. The resulting formula $\neg \phi$ is converted to a Büchi automaton $A_{\neg \phi}$, called a "never claim" in the parlance of Spin, that recognizes precisely this set of bad behaviors. The Spin system then takes the automata $\mathcal{M}$ and $A_{\neg \phi}$ as input and reduces model checking to an automata-theoretic problem as described in [VW86] by constructing (on-the-fly) and searching the resulting product automaton. If Spin finds a trace accepted by this automaton, then the trace is a counterexample to $\phi$ that is accepted by $\mathcal{M}$ and it explains how the system allows the bad behavior. Alternatively, if Spin succeeds in showing that no such traces exist (by exhaustively showing that there are no acceptance cycles in the product automaton), then it has succeeded in showing $\mathcal{M} \models \phi$.

We now illustrate how Spin is used to verify the first requirement described in Sect. 2.3. This requirement states that the signature architecture must authenticate a user before the user generates a signature. In our Z specification, we formalized this using event predicates that characterized the effect of events. Our PROMELA model is more operational and we can formalize predicates not only in terms of the global system state, but also in terms of the state of the individual processes, e.g., their program counters. This results in direct, albeit lower-level definitions of predicates like In, Out, and Sign, which we defined previously.

The following are our PROMELA definitions (written using C-preprocessor notation) of these three predicates.

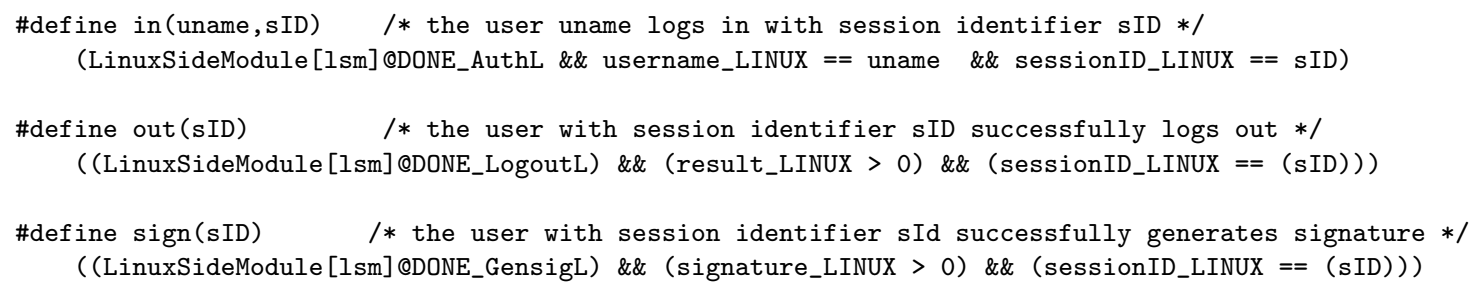

In these definitions, we reference labels (using @) in our PROMELA model to formalize that processes have reached certain points in their execution, and we use predicates on variables to express conditions on the system state. For example, consider the predicate in, which formalizes the state reached after a user executes the login function. It consists of three conjuncts which formalize that the Linux-side module has: (1) reached the step labeled Done_AuthL, indicating that authentication has completed (see Fig. 10); (2) the user being authenticated is uname; and (3) the session identifier returned is $S I D$. Note that authentication can fail, in which case $s I D$ is zero. The other predicates are formalized similarly.

Now we can formalize (R1) using these predicates: each signature generated with a session identifier $s$ must be preceded by a login of a user $u$, who is assigned $s$ and has not logged out in the meantime. We express this as

$$
\forall s: \text { session. }(\exists u: \text { user. }(\text { in }(u, s)) \text { before } \operatorname{sign}(s)) \wedge \square(\operatorname{out}(s) \rightarrow((\exists u: \text { user.in }(u, s)) \text { before } \operatorname{sign}(s))) \text {. }
$$

The first conjunct states that whenever a signature is generated for a session with identifier $s$, it must be preceded by some user $u$ who logs in and is allocated $s$. The second states that, after each logout that terminates the session $s$, then again any signature with $s$ must be preceded by a login allocating $s$. Here user ranges of the set of all user names and session over the set of valid session identifiers (i.e., those greater than zero), which result from a successful login. This formula formalizes (R1) as any signature must be preceded by a successful login, and a successful login must also occur after the last logout preceding a signature. 


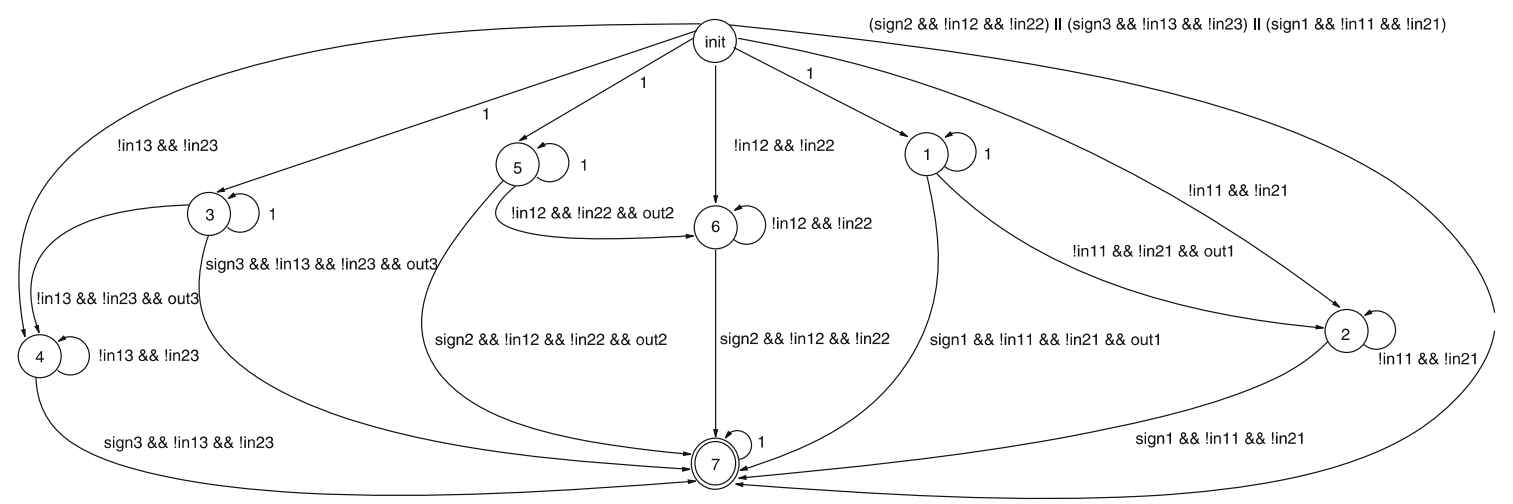

Fig. 12. Büchi automaton generated for never claim for requirement 1

The above is not yet a formula of future-time LTL, to which Spin is limited. To begin with, "before" is not a standard LTL operator (see Appendix A for a definition of LTL). However it can be expressed using the "weak until" modality $\mathcal{W}$, where $A$ before $B$ is defined as $(\neg B) \mathcal{W} A$. Substituting this definition, Equation (1) becomes

$\forall s:$ session. $(\neg \operatorname{sign}(s) \mathcal{W}(\exists u:$ user.in $(u, s))) \wedge \square(\operatorname{out}(s) \rightarrow(\neg \operatorname{sign}(s) \mathcal{W}(\exists u: u \operatorname{ser} . \operatorname{in}(u, s))))$.

Finally, we must eliminate the two quantifiers over sets. Since these sets are finite, we can replace the universally quantified formula by finitely many conjuncts and the existentially quantified formulas by finitely many disjuncts. In particular, the formula $\forall s$ : session. $P(s)$ is expanded to $P\left(s_{1}\right) \wedge P\left(s_{2}\right) \cdots \wedge P\left(s_{n}\right)$, where $s_{1}, \ldots, s_{n}$ are the finitely many model representatives of valid session identifiers and the dual replacement is made in the existential case. So, for example, for a scenario with 3 sessions and 2 users, Eq. (2) becomes

$$
\begin{aligned}
& \neg \operatorname{sign}\left(s_{1}\right) \mathcal{W}\left(\operatorname{in}\left(u_{1}, s_{1}\right) \vee \operatorname{in}\left(u_{2}, s_{1}\right)\right) \wedge \square\left(\operatorname{out}\left(s_{1}\right) \rightarrow\left(\neg \operatorname{sign}\left(s_{1}\right) \mathcal{W}\left(\operatorname{in}\left(u_{1}, s_{1}\right) \vee \operatorname{in}\left(u_{2}, s_{1}\right)\right)\right)\right) \wedge \\
& \neg \operatorname{sign}\left(s_{2}\right) \mathcal{W}\left(\operatorname{in}\left(u_{1}, s_{2}\right) \vee \operatorname{in}\left(u_{2}, s_{2}\right)\right) \wedge \square\left(\operatorname{out}\left(s_{2}\right) \rightarrow\left(\neg \operatorname{sign}\left(s_{2}\right) \mathcal{W}\left(\operatorname{in}\left(u_{1}, s_{2}\right) \vee \operatorname{in}\left(u_{2}, s_{2}\right)\right)\right)\right) \wedge \\
& \neg \operatorname{sign}\left(s_{3}\right) \mathcal{W}\left(\operatorname{in}\left(u_{1}, s_{3}\right) \vee \operatorname{in}\left(u_{2}, s_{3}\right)\right) \wedge \square\left(\operatorname{out}\left(s_{3}\right) \rightarrow\left(\neg \operatorname{sign}\left(s_{3}\right) \mathcal{W}\left(\operatorname{in}\left(u_{1}, s_{3}\right) \vee \operatorname{in}\left(u_{2}, s_{3}\right)\right)\right)\right) .
\end{aligned}
$$

The negation of this formula constitutes our never claim, which we automatically convert into a Büchi automaton with 8 states. For this we use the tool LTL2BA [GO01]. Figure 12 shows the resulting automaton where, to save space, we have abbreviate $\operatorname{sign}\left(s_{1}\right)$ as $\operatorname{sign} 1, \operatorname{in}\left(u_{1}, s_{2}\right)$ as $\operatorname{in} 12$, etc. Although it would not be easy to specify an automaton like this by hand, it is not too difficult to understand how it works. Roughly speaking, the automaton consists of three parts-right, center, and left-which handle the cases of signatures produced with the first, second, and third session identifier respectively. Consider, for example, the scenario where:

1. the system allows a user to $\log$ in with session identifier 1 ,

2. session 1 is terminated by a log out,

3. some number of steps follow without the session identifier 1 being reassigned by a log in, and

4. a signature is produced in a session with identifier 1.

This scenario, representing a violation of (R1), is accepted by this automaton. Step 1 corresponds to a transition from the initial state to state 1 (a transition labeled 1 can always be taken, including during a login). Actions taken by the system during this session correspond to the self-loop on state 1 (it is immaterial what these actions are, e.g., perhaps a signature is generated or perhaps not). Step 2, the $\log$ out, corresponds to the transition from state 1 to state 2 . Step 3 corresponds to the self-loop on state 2 . Finally step 4 corresponds to the transition from state 2 to the final state 7 , an accepting state.

Spin verifies that our model satisfies this property (i.e., no system trace satisfies the never claim) in 45 minutes of computation time. ${ }^{6}$ In doing so, it builds a product automaton with over 17 million states and searches almost 60 million transitions. Note that while model checking itself is completely automatic, the model checker may fail to terminate or may exhaust memory. This was often the case in our work and it required considerable interaction to obtain a successful run. We will return to this point in the comparison.

\footnotetext{
${ }^{6}$ All verification times measured in this paper are on a 4 Ghz Intel P4 workstation with 4 GByte RAM running Linux.
} 


\begin{tabular}{lll}
\hline Measurement & HOL-Z & PROMELA/Spin \\
\hline Model Variants & 1 & 4 \\
Model Size & 550 lines & 647 lines (average) \\
Model Bounds & unbounded & 2 users, 2 sessions \\
Property Size & 50 lines & 53 lines \\
Proof Size & 3662 lines & none \\
\hline Property Specification Time & 2 days & 7 days \\
System Modeling Time & 12 days & 17 days \\
Verification Time & 19 days & (included above) \\
Proof Checking Time & 12 minutes & 96 minutes \\
Total Time & 33 days & 24 days \\
\hline Expert Input Required & $60 \%$ & $15 \%$ \\
\hline
\end{tabular}

Fig. 13. Statistics on the two verifications

The formalization and verification of the second requirement follows the same pattern. However, the third requirement necessitated a different approach. This requirement involves counting: the signature architecture must generate only one hysteresis signature per authentication. While it is straightforward to count up to some finite bound in LTL, it requires using the next-time modality. This means that optimizations, like partial-order reduction [Pe196], which play an important role in making model checking feasible, are no longer sound. As a result, our attempts at model checking this way failed. We took an alternative approach and modified our model, adding an array of counters, one for each session identifier, that tracks the number of signatures generated for each session. We then added a monitor process that runs in parallel and checks that each counter in the array is either set to zero or one. Afterwards we used Spin to automatically verify that this assertion holds for all reachable system states.

\section{Comparison}

In this section, we compare the two different approaches we have taken to modeling and verification. While there have been other general investigations of theorem proving versus model checking (see Sect. 6) and considerable work on their integration, there appear to be few studies that examine their relationship concretely on an in-depth case study. We take up this challenge here and make both quantitative and qualitative comparisons between our two formalizations. The results, we believe, help shed light on the relative strengths and weaknesses of the different approaches.

Note that any such comparison must be made and interpreted with care. The conclusions can differ considerably depending, for example, on the expertise of those carrying out the verification, the specific formalisms and tools used, what is actually measured, and the problem chosen for the comparison. [BK91] contains an in-depth discussion of the difficulties involved here. To ensure an accurate quantitative comparison, we have kept statistics on both verification efforts (the times spent are estimates) and also ensured that each verification was made on an equal footing. Both verifications were carried out by a team consisting of an expert in the formal method (namely, a researcher who both understands the theory behind the formal method and has practical experience in its use) and an engineer with limited initial knowledge of the formal method.

Figure 13 summarizes the quantitative differences between the two approaches. We explain these figures below, as well as qualitative differences not captured by these metrics.

\subsection{Size}

In HOL-Z, we built one system model, of 550 lines, against which we verified all three properties. In PROMELA, we built an initial system model, which we adapted afterwards for each of the three properties. The 647 lines of specification is the average size of the four models created. Despite the fact that the HOL-Z model differs substantially from the PROMELA models, they are all of roughly similar size. This stems from the fact that the HOL-Z model is more detailed than the PROMELA models in some respects and more abstract in others. For example, HOL-Z state schemas are more detailed since they define not only data types, but also invariants. On the 
other hand, HOL-Z operation schemas are typically smaller as they abstractly specify the relationship between states, rather than the sequence of operations used to change states.

For HOL-Z, property size measures the number of lines used to specify the properties and the event predicates used in their definitions. For PROMELA, it is the number of lines used to specify the LTL formulas, the auxiliary predicates, and the changes to the model needed to specify the requirements (including the introduction of a monitor process for the third requirement). The LTL formulas for the first two requirements are quite compact, at least before we expand the set quantifiers. ${ }^{7}$ This need not generally be the case: there are temporal requirements whose LTL formalization are non-elementary larger than their formalization in higher-order logic. ${ }^{8}$ Finally note that Spin works with the Büchi automata generated from the LTL formulas, and the automata can be exponentially larger. While the automata are generated automatically and it is not actually necessary for users to ever examine them (as we did in Sect. 4.7), we found that this was helpful in checking that our LTL formulas actually captured the intended requirements.

\subsection{Time}

More time was spent in the theorem-proving approach than in the model-checking approach. The main difference is due to the fact that model checking is automatic as opposed to interactive (the 19 days reflects the time spent interacting with the theorem prover). Folk wisdom is that, because of automation, model checking is much less time consuming than theorem proving. While this is indeed the case for the verification time itself, the overall time reduction, about $27 \%$, is not so significant.

However, the numbers point only indirectly to what is probably the most interesting difference: how the time was spent. With Spin, once a model and a property are specified, the verification effort is focused on simplifying the problem so that the model checker terminates. In our case, this involved tuning the sizes of the different finite domains as well as introducing abstractions and other simplifications. For example, to reduce verification times, we found it necessary to annotate our model with information (using PROMELA's atomic statement) on which sequences of steps can safely be executed atomically, i.e., without interleaving steps from other processes. Moreover, as previously noted, although it is possible to model the requirement (R3) using a never claim, the resulting verification consumed too much computer memory and thus required a different modeling approach. The time spent on these activities (which also includes waiting for runs to fail) was substantial and is reflected both in the increased time taken for system modeling and for property specification.

Note that these efforts are quite different from those required for verification in HOL-Z. Our HOL-Z verification was based on only one model, the general system model. We neither had to work out any abstractions or restrictions in advance nor to make subsequent changes during verification. Hence the specification time was shorter. In return, substantially more time was required for verification. Although some of this time was spent pushing low-level proof details through the Isabelle system, as explained in Sect. 3.5, much of it concerned discovering, formalizing, and proving auxiliary system invariants, which were required to prove the properties of interest.

Although discovering and proving invariants is a more time-consuming activity than (PROMELA) model simplification, it is also a more insightful one. Many of the invariants are interesting in their own right as they lead to a better understanding of why the architecture actually works. Moreover, in our work, they also led to our discovering problems in our original formalization of Hitachi's requirements. For example, a direct formalization of the first requirement (that signature generation requires a prior login) overlooks the fact that the login session must still be valid, that is, there cannot be a logout between these events. We originally formalized and verified this weaker statement [i.e., the statement that arises from omitting the last conjunct in the theorem statement in Sect. 3.4 or equivalently the second conjunct in Eq. 1] in Spin. Only when working out the invariants in HOL-Z did we realize that the stronger theorem was actually intended and held. Such specification errors can of course be found by careful review, but our experience is that they are much more likely to be made during model checking where users are only confronted with the direct consequences of what they specify.

\footnotetext{
7 We have counted their size after expansion, e.g., counting Equation 3 as 3 lines. Note then that the size of the LTL specification is a function of the size of the finite domains involved and had we been able to model check larger models, our specifications would have also been larger. This is a general problem with specification languages based on propositional logic.

8 The first-order theory of linear orders $F O[<]$ with unary predicates, whose formulas can be linearly embedded in higher-order logic, has a non-elementary worst-case complexity. In particular, there are families of formulas whose Büchi automata have non-elementary many states with respect to the formula length.
} 


\subsection{Expertise needed}

In both case studies, expert input was needed, albeit to a different degree and in different places. In both approaches, it was possible for an engineer with limited initial knowledge of the formal method to build the first model after receiving some training for the task. However, for the HOL-Z model, an expert review and restructuring of the model was needed. Finding suitably abstract formulations in $\mathrm{Z}$ appears to require more expertise than finding "natural" formulations in PROMELA, which was perceived as a kind of programming language.

In contrast, in the Spin case study, most of the expert help required was in formulating properties. This turned out to be surprisingly tricky. Temporal formulas like that of Equation (2) are difficult for novices to formalize, as familiarity with LTL idioms (e.g., specification patterns as in [DAC99]) is helpful for translating statements about the past to those about the future. Also problematic is validating that a given LTL formula actually captures the intended requirements. We did not have these problems with the HOL-Z formalization as the use of the more expressive logic of HOL-Z allowed us to directly quantify over time and make standard comparisons on time points, which is simpler and more intuitive than the use of LTL.

Of course, capturing requirements in LTL does offer certain advantages over their formalization as predicates over traces in HOL. The LTL specifications tend to be more concise than their HOL counterparts. Moreover, in the case of first-order LTL, where verification is no longer decidable, the syntactic form of the temporal formulas may suggest useful proof strategies and inference rules for theorem proving; [CS05, MP91] provide examples of this. Hence an interesting possibility would be to find a middle ground, between these LTL and HOL. One alternative here is to use a more expressive (but still elementary decidable) temporal logic like the real-time extension of LTL proposed in [AH94]. This logic provides constructs for binding variables to time points and making explicit comparisons between time points; this fits well with the kinds of temporal constructs that we used when specifying properties in HOL. Another alternative is to work with a suitable embedding of temporal operators directly within higher-order logic. This allows us to move between these different specification paradigms during specification and theorem proving as well as derive temporal idioms and formally reason about the relationship between different formalizations of requirements. We sketch this option and provide examples in Appendix B.

Finally, the most substantial difference in terms of the expertise required concerned the verification itself. Model checking is push button, but only in theory. In practice, some expert input was required in restructuring and tuning the model so that Spin would terminate. In contrast, the HOL-Z study required considerable handson work by the expert in order to complete some of the proofs. This is reflected by the $60 \%$ expert contribution reported in Fig. 13.

\subsection{What was formalized and proved}

Finally, the numbers given do not reflect that there were substantial differences, as well as similarities, in what was modeled and verified. Any model of the signature architecture worthy of the name must capture both dataoriented and process-oriented aspects at some level of abstraction. The HOL-Z model does this by providing a data-oriented interface model that specifies the different components of the system state and a relational formalization of the state-transition operations. The resulting model is extended to a simple process model with an interleaving trace semantics. This is in contrast to the PROMELA model where the operational and communication behavior of the interface functions is spelled out concretely, albeit on simplified data domains. SPIN constructs from this a transition system, which has again an interleaving trace semantics. So at first glance we see both similarities (trace semantics) and differences (specification styles and what was actually modeled). We consider below the main differences in more detail as well as their implications.

First, in HOL-Z, we were able to directly model the relevant data domains in their full generality, rather than settling for some finite approximation. This means we did not need to bound, a priori, the size of domains like the set of users, their passwords, session identifiers, and the like. This is in contrast to the PROMELA model, where all data domains were simplified to small finite domains to support model checking, as explained in Sect. 4.4. The ability to model and reason about infinite domains is a standard benefit of using a rich logic like HOL-Z. Our HOL-Z model is both more general and the theorems proven are significantly stronger.

Second, the two models differ in the way that concurrency and communication are expressed. The PROMELA language is based on the notion of processes which may run in parallel, and communicate either synchronously or asynchronously over communication channels. So direct support for concurrency and communication are built into the language. This is not the case for HOL-Z as the focus is on data modeling, not concurrent process modeling, and hence both concurrency and communication must be explicitly modeled within the language. We 
introduced concurrency in our HOL-Z model only at the top level of the architecture in coarse-grained client and server steps and we modeled communication by shared schema variables. As the communication model in the architecture was fairly simple, this top-level synchronization was adequate.

One modeling consequence of this is that PROMELA's bias towards process-oriented modeling led naturally to a model where an attacker is explicitly formalized and whose interface calls are interleaved with those of honest users. This formalization, which has its roots in protocol analysis, has the advantage of explicitly modeling the powers of the attacker for disrupting the system. From the HOL-Z perspective, concurrency is expensive during modeling and even more so during theorem proving. The architecture decomposition theorem is used to make the possible combinations explicit and constructing the resulting proofs would become much more difficult if fine-grained concurrency were allowed between the components (which is why model checking is the preferred approach in such cases, when it is possible). The HOL-Z model shows another way to proceed: this model makes no constraining assumptions on the clients at all-neither in the order of operations nor in the data sent along DARMA (as long as its types are respected) -and therefore identifies the client with the attacker.

Finally, as noted above, although the two approaches specify the same interface functions, the HOL-Z specification is declarative, while the PROMELA specification is operational. The operational approach, by its very nature, involves commitments to data types and concrete procedures for data manipulation. In contrast, HOL-Z does not require such commitments and this leaves us considerably more flexibility in how the architecture can be refined and for exploring changes. As an example, in the Hitachi architecture, a user may only log in once before logging out again, i.e., a user may be associated with only one session. However, an alternative architecture is one that supports multiple sessions per user. Modeling these kinds of changes in our architecture is trivial. Here, we can specify this alternative simply by deleting the second constraint in the predicate part of the session manger schema (Sect. 3.2), which requires that each user identifier is associated with at most one session identifier. In this case, almost all of the system invariants proven go through, unchanged.

\section{Related work and conclusions}

It has become a tradition to call a questionable common belief a "myth" [Ha190, BH95]. Of course, it can be questioned to what extent our case study represents a valid criticism of the myth of the substantially higher effectiveness of model checking versus theorem proving. Understood as strong myth ("model checking is always more effective than theorem proving"), our work constitutes a counterexample. Understood as a weak myth ("model checking is usually substantially more effective than theorem proving"), only a large-scale study could validate or refute it and such a study has not been undertaken yet. But even against the weak myth, our case study suggests that one must carefully distinguish the relative strengths and weaknesses. Examples we have seen that highlight the relative strengths of theorem proving include clearer and more general specifications, stronger theorems, and increased confidence gained by formalizing and proving system invariants. With respect to the last point, we note that the absence of counter-examples for a property says relatively little about the adequacy of its formalization (see Sect. 5.2 for an example of this problem).

To the best of our knowledge, there have been no other comparisons between model checking and theorem proving, with the exception of [Gup92], focusing on hardware verification and dating back nearly 15 years. Most research involving the two paradigms has focused on their combination: how to best build model checkers and other decision procedures into theorem-proving tools. The main applications are to decide subproblems that fall into decidable subtheories, e.g., propositional logic and arithmetic [BF00, BM88, FORS, Nor, $\left.\mathrm{SJO}^{+} 05\right]$, and to use model checkers for problems that can be reduced to finite-state systems, e.g., using abstraction [MN95, $\mathrm{ORR}^{+} 96$, RSS95]. The two paradigms can be combined too in the sense that model checking (working with finite abstractions) can be employed at an early stage to detect flaws, in order to gain confidence in a system's correctness, before turning to theorem proving, e.g., as suggested in $\left[\mathrm{RSG}^{+} 00\right]$.

In contrast, there have been many comparisons made between other formal methods and verification approaches. Examples include comparisons between different specification languages [ABL86], between different theorem provers [BK91], and between different automated analysis techniques (including model checking, static analysis, and testing) $\left[\mathrm{ACD}^{+} 99, \mathrm{Cor} 96, \mathrm{BDG}^{+} 04\right]$. The domain we have chosen, architecture modeling and verification, is one that has been extensively studied before using both data-oriented and process-oriented formal methods, e.g. [AAG95, CAB ${ }^{+}$98, JS00, SG96, WVF97]. However, the focus of these other studies has been different, namely showing the suitability of the different individual formal methods for the verification task.

Our work also sheds light on the suitability of using $\mathrm{Z}$ and the associated HOL-Z environment for formalizing and verifying architectures that combine data-oriented and process-oriented aspects. It should be noted in this 
regard that our modeling and verification of the signature architecture is the largest case study made to date using HOL-Z. Previous case studies also include a security architecture (for controlling access to a repository) [BW03], but there the emphasis was on data refinement, rather than the verification of temporal properties of system runs. The studies are complementary in that together they illustrate how HOL-Z can be used to formalize, verify, and refine architectures at different levels of abstractions, covering both data-oriented and process-oriented aspects.

There are a number of avenues open for future work. To begin with, the theorems we proved by model checking are weaker than those proved using HOL-Z. In some situations however it may be possible to verify the correctness of the abstractions used, i.e., that the verification of the small finite model used entails the verification of the corresponding infinite state system whose state variables range over infinite data domains. Techniques based on data independence, such as those of [Low98, RB99], may help automate this task.

Another direction concerns the way we modeled cryptographic functions. In the HOL-Z model, cryptographic functions for hashing and signing were simply treated as uninterpreted function symbols, specified just by their type. This leaves open all possible implementations, faithful or not to the standard cryptographic requirements for such functions. In the PROMELA model, we had to take the other extreme and commit to concrete computable functions. Our formalization has the property that certain actions, like guessing values, are impossible as opposed to being highly improbable, which is the case in actual cryptography. As the properties we examined were possibilistic rather than probabilistic, this was not a problem. However, it would be interesting to investigate whether cryptographically sound abstractions such as those of [Can01, BPW03, $\left.\mathrm{SBB}^{+} 06\right]$ could be usefully employed in this setting.

Finally, while we learned much ourselves from this comparative case study, and hope that others can also profit from it, it is of course only one data point documenting the issues and tradeoffs involved. Our case study combined both data-oriented and process-oriented aspects, but most of the complexity was in the data-modeling side and, as noted in Sect. 5.4, the HOL-Z communication model was coarse grained. For this combination, there were striking benefits from using the HOL-Z approach. But for a system with simpler operations and more complex process interaction, the conclusions might be quite different. An example of this would be architectures whose complexity is dominated by the communication protocols employed: here we would expect model checking to have the upper-hand. Said another way, the tradeoffs are not absolute, but relative to the problem under consideration. Additional comparative case studies could contribute to our understanding of this relationship and to the development of refined guidelines for the use of different formal methods.

\section{Acknowledgments}

We thank Jean-Raymond Abrial, Achim Brucker, Christoph Sprenger, Felix Klaedtke, and Ernst-Rüdiger Olderog for helpful discussions on the contents of this paper. We thank Shinji Itoh, who wrote Hitachi's specification document and answered our questions about it. Finally, Kunihiko Miyazaki thanks the Japanese National Institute of Information and Communications Technology (NICT) for partially supporting this work.

\section{Appendix A: Linear temporal logic}

Syntax. Let a set of assertions (also called state formulas) be given. A (full) LTL formula is built from assertions using the Boolean connectives $\neg$ and $\wedge$ and the temporal operators $\bigcirc$ (Next), $\ominus$ (Previous), $\mathcal{U}$ (Until), and $\mathcal{S}$ (Since). Future-time LTL is the subset of full LTL where the only temporal operators are $\bigcirc$ and $\mathcal{U}$. Note that we will also employ additional propositional constants ( $\mathbf{T}$ and $\mathbf{F}$ ) and connectives (defined as standard) as well as the following defined temporal operators:

$$
\begin{aligned}
& \diamond p \quad=\mathbf{T} \mathcal{U} p \quad \text { (sometime, eventually) } \\
& \square p \quad=\neg \diamond \neg p \quad \text { (always, henceforth) } \\
& p \mathcal{W} q=\square p \vee(p \mathcal{U} q) \quad \text { (unless, weak until) }
\end{aligned}
$$

Semantics. Let a set of states $\sigma$ be given. Semantically, each assertion defines a predicate over states of type $\sigma \rightarrow$ bool. A model is an infinite trace $t$ of type nat $\rightarrow \sigma$. Given a model $t$, we define the satisfaction of a formula 
$p$ at position $j \geq 0$, written as $(t, j) \models p$, by cases.

$$
\begin{array}{lll}
(t, j) \models p & \Leftrightarrow p(t(j)) & \text { (assertion) } \\
(t, j) \models \neg p & \Leftrightarrow \neg((t, j) \models p) & \text { (negation) } \\
(t, j) \models p \wedge q & \Leftrightarrow(t, j) \models p \wedge(t, j) \models q & \text { (conjunction) } \\
(t, j) \models \bigcirc p & \Leftrightarrow \quad t, j+1) \models p & \text { (next) } \\
(t, j) \models p \mathcal{U} q & \Leftrightarrow \exists k \geq j .(t, k) \models q \wedge(\forall i . j \leq i<k \Rightarrow(t, i) \models p) & \text { (until) } \\
(t, j) \models \ominus p & \Leftrightarrow j>0 \wedge(t, j-1) \models p & \text { (previous) } \\
(t, j) \models p \mathcal{S} q & \Leftrightarrow \quad \exists k \leq j .(t, k) \models q \wedge(\forall i . k \leq i<j \Rightarrow(t, i) \models p) & \text { (since) }
\end{array}
$$

Given a Kripke structure $K$, we write $K \models p$ iff $(t, 0) \models p$ for all $t$ of $K$.

\section{Appendix B: HOL requirements in an LTL style}

In Sect. 5.3 we observed that LTL has advantages over HOL in terms of conciseness and inference rules. We now return to this point and show that it is possible to have the best of both logics via an embedding of LTL within HOL. Note that all of the definitions and equivalence proofs given here have been checked in Isabelle/HOL.

Recall from Sect. 3.4 that event predicates constitute abstract actions. Given a Kripke structure $K=($ Init, Next $)$, we can transform it into a Kripke structure suitable for reasoning about actions, a so-called action Kripke structure, by the bijection $K_{A}$ of type $(\mathbb{P} \sigma \times(\sigma \leftrightarrow \sigma)) \rightarrow(\mathbb{P}(\sigma \times \sigma) \times((\sigma \times \sigma) \leftrightarrow(\sigma \times \sigma)))$ defined by

$$
\begin{aligned}
K_{A}(\text { init }, \text { trans }) \equiv( & \left(\left\{\left(s, s^{\prime}\right) \mid s \in \text { init } \wedge\left(s, s^{\prime}\right) \in \text { trans }\right\},\right. \\
& \left.\left\{\left(\left(s, s^{\prime}\right),\left(t, t^{\prime}\right)\right) \mid s^{\prime}=t \wedge\left(s, s^{\prime}\right) \in \text { trans } \wedge\left(t, t^{\prime}\right) \in \text { trans }\right\}\right) .
\end{aligned}
$$

In contrast to $K$, the assertions of $K_{A}($ Init, Next $)$ are predicates over pairs of states, i.e., actions from our HOL-Z model.

It is now a simple matter to translate the HOL formalizations of our three requirements into LTL, which we illustrate here with (R1). One such formulation is

$$
K_{A}(\text { Init, Trans }) \models \square(\operatorname{Sign}(\text { uid }) \longrightarrow(\neg \text { Out (uid) } \mathcal{S} \operatorname{In}(\text { uid }))),
$$

which says that "every time the user uid produces a signature, the user has previously logged in, and not logged out since then". It is not difficult to prove that this is equivalent to our HOL formulation given in Section 3.4. Unfolding this definition yields

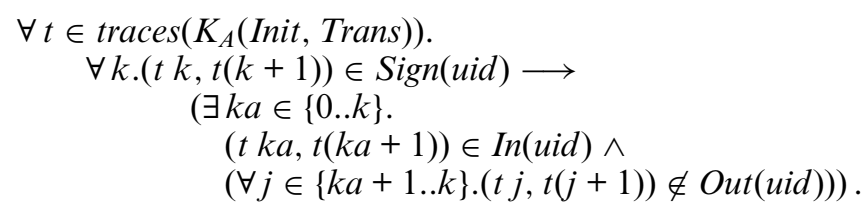

Although this is not identical to our original formulation, it is equivalent given that the actions Sign, In and Out are disjoint. Moreover, using standard equivalences (see [MP92]), it is possible to convert this formula into a future-time LTL formula. In particular, using

$$
\square(P \longrightarrow(Q \mathcal{S} R)) \Leftrightarrow \neg P \mathcal{W} R \wedge \square(\neg Q \longrightarrow \neg P \mathcal{W} R))
$$

we can rewrite and simplify (4) to

$$
\left.K_{A}(\text { Init, Trans }) \models \neg \operatorname{Sign}(\text { uid }) \mathcal{W} \operatorname{In}(\text { uid }) \wedge \square(\text { Out(uid }) \longrightarrow \neg \operatorname{Sign}(\text { uid }) \mathcal{W} \operatorname{In}(\text { uid })\right)
$$

At this point, the connection to the LTL formalization of (R1) in the Spin case study (see Section 4.7, Equation (2)) becomes apparent: both have the identical temporal structure. The only differences concern the way the particular HOL-Z actions (versus PROMELA assertions) and associated information (user identifiers and session identifiers) are formalized.

The above shows one use of HOL specifications in a temporal style: we can relate higher-order logic specifications to those in weaker logics like LTL. We conclude here by describing a second use: the LTL embedding can serve as the basis for deriving useful proof rules. We given an example of this, which also sheds some light on our observation in Sect. 3.5 that most of our effort in HOL-Z verification was devoted to local reasoning about preconditions and postconditions of operations. 


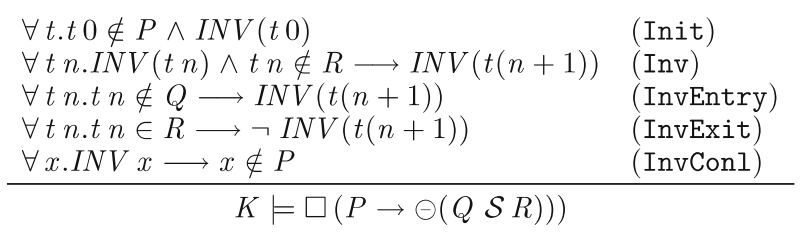

Fig. 14. Rule SafeSince

An alternative, equivalent, formalization of (R1) is

$K_{A}($ Init, Trans $) \models \square \operatorname{Sign}($ uid $) \longrightarrow \ominus(\neg$ Out (uid) $\mathcal{S} \operatorname{In}($ uid $))$.

Figure 14 presents a derived rule for reasoning about specifications of this form, motivated by the inference rule SAFE of [MP91, page 9]. We have used this rule to reason about temporal formalizations of the three requirements (R1) - (R3). ${ }^{9}$ In this rule, $t$ ranges over traces and $n$ ranges over natural numbers. Note that when interpreted over the traces of an action Kripke structure, $t n$ refers to a pair of states $(t n, t(n+1))$.

When applied, the premises of the rule often suggest an appropriate invariant $I N V$. For example, when applied to verify (5), $P$ is instantiated by $\operatorname{Sign}($ uid),$Q$ by $\neg \operatorname{Out}($ uid), and $R$ by $\operatorname{In}($ uid). From the structure of the premises we can easily guess the invariant $I N V$ : the user uid should not be authenticated at the beginning of the state transition. This invariant can be easily expressed in the terms of the system model: uid must not be in the domain of the initial session table. Applying SafeSince with this instantiation reduces the overall burden to prove global (R1) into the following proof obligations, all of which are local.

1. At the beginning, the generation of a signature is not possible and no user is authenticated (Init).

2. If a user is not authenticated and no login occurs, he remains unauthenticated (Inv).

3. If a logout occurs, i.e. $\neg \neg$ Out(uid), the user should not be authenticated (InvEntry).

4. If a login occurs, the user should be authenticated (InvExit).

5. If the user is not authenticated, no generation of a signature is possible (InvConcl).

The proof of SafeSince itself is a routine induction over the position in a trace. In contrast, the proof of (InvConcl) is two orders of magnitudes larger and substantially more complex. Discharging the other proof obligations is easy since they only use abstract system operations themselves; thus, they profit from the abstraction of the underlying refinement.

\section{Appendix C: A sample proof in HOL-Z}

In this appendix, we present an example of theorem proving in HOL-Z. As HOL-Z expressions are represented using Isabelle-specific concepts, we begin with a brief overview of Isabelle [NPW02].

\section{C.1. Proofs in Isabelle}

Isabelle is a logical framework, which means it provides a meta-logic for representing other logics, like HOL. Isabelle's meta-logic is based on intuitionistic implication $\Longrightarrow$ and higher-order quantification $\Lambda$.

An Isabelle proof state is a formula in Isabelle's metalogic that is logically valid, i.e., a theorem. To prove a goal $\phi$, one converts it into a proof state: the theorem $\phi \Longrightarrow \phi$. Proof proceeds by iteratively transforming the premise of this theorem using tactics, which are programs that implement proof-state transformations. A tactic refines the premises of this topmost implication - called subgoals - into other formulas. Subgoals have the general form

$$
\bigwedge x_{1} \ldots x_{m} \cdot\left[A_{1} ; \ldots ; A_{n}\right] \Longrightarrow A \text {. }
$$

\footnotetext{
${ }_{9}$ In the case study we verified non-temporal, higher-order formalizations, as we reported on in Sect. 3.4. Afterwards completion of the case study, in order to better understand the relationships between the two models, we carried out the temporal formalization in HOL and experiments using derived (temporal) proof rules. The statistics reported on in Section 5 are for the original case study, and do not include the post-hoc reformulation and experiments.
} 
This (meta-)formula states that $A$ follows from the assumptions $A_{1}$ through $A_{n}$, for arbitrary $x_{1}, \ldots, x_{m}$. A tactic may transform a subgoal into the formula True, at which point it is discharged by deleting it from the premises. A proof state without subgoals is closed. In this case, the goal can be extracted as a theorem.

\section{C.2. An example}

We present here a concrete example of a HOL-Z proof of one of our 173 theorems. We prove a non-trivial system invariant: the signature log grows monotonically during system execution (i.e., along each possible execution trace). The signature_log is modeled as a sequence and sequences are modeled in $\mathrm{Z}$ as functions from natural numbers to values, and therefore as sets of pairs (of natural numbers and values). Hence this invariant can be formalized using set inclusion, as follows.

$$
\begin{aligned}
& \bigwedge t \in \text { Traces }, i \in N \\
& \Longrightarrow \operatorname{dom}((t \text { i).signature_log }) \subseteq \operatorname{dom}(t(i+1) \text {.signature_log })
\end{aligned}
$$

Let claim be the string representation in Isabelle of the above formula.

Our proof proceeds as follows. We first initialize the proof state with claim, the formula we wish to prove.

val prems = goalw Analysis.thy [] claim;

Next we apply three proof-state transformations. The main transformation is the application of the lemma State_Transition_Cases, which represents the right-to-left application of the architecture decomposition theorem discussed in Section 3.5. The other two commands perform elementary simplifications.

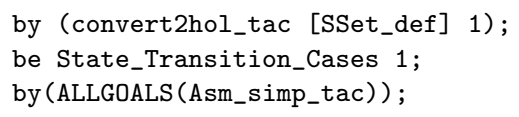

Applying these steps in Isabelle results in the proof state displayed in Fig. 15. Hence we have reduced showing that our invariant holds for all possible transitions to showing that it holds in each of the four cases described by the decomposition theorem, i.e., that it is preserved by each of the four ways that the system may evolve.

We proceed now to the first subgoal, which states that the invariant is preserved after the system takes a parallel AuthenticateUser-step, both on the client and the server side. The key fact needed, which we establish separately as a lemma (named AuthenticateUserL_inv_state_components), is that AuthenticateUserL does not change the access controller's state.

$\vdash \quad$ AuthenticateUser $L \Rightarrow$ (signature_log' $=$ signature_log $\wedge$ access_control_list ${ }^{\prime}=$ access_control_list $\wedge$ pri_key_list' $=$ pri_key_list $)$

It is worth noting that $\mathrm{Z}$ schema reference AuthenticateUserL is just a pretty-printed version of the (less pretty) term:

$$
\begin{aligned}
& \text { AuthenticateUserL(Authentication, Command, Logout_ID, Result, SessionID!, Signature, } \\
& \text { Signature_generation_hmg, Signature_generation_sid, User_authentication_pw, } \\
& \text { User_authentication_uid, access_control_list, access_control_list' }{ }^{\prime} \text { password?, } \\
& \text { pri_key_list, pri_key_list', session_IDs, session_IDs', session_table, } \\
& \text { session_table', signature_log, signature_log' }{ }^{\prime}, \text { username? }^{\prime} \text {. }
\end{aligned}
$$

Thus, in the HOL-representation, the implicit binding of the $\mathrm{Z}$ notation is made explicit, which allows for arbitrary renamings of state components throughout the proof as needed (renaming corresponds to $\alpha$-conversion in Isabelle). This expansion of schemas to predicates has occurred in Fig. 15.

We apply this lemma using a HOL-Z tactic zdtac, which "lifts" the lemma to the binding structure in the first subgoal.

by(zdtac AuthenticateUserL_inv_state_components 1); 


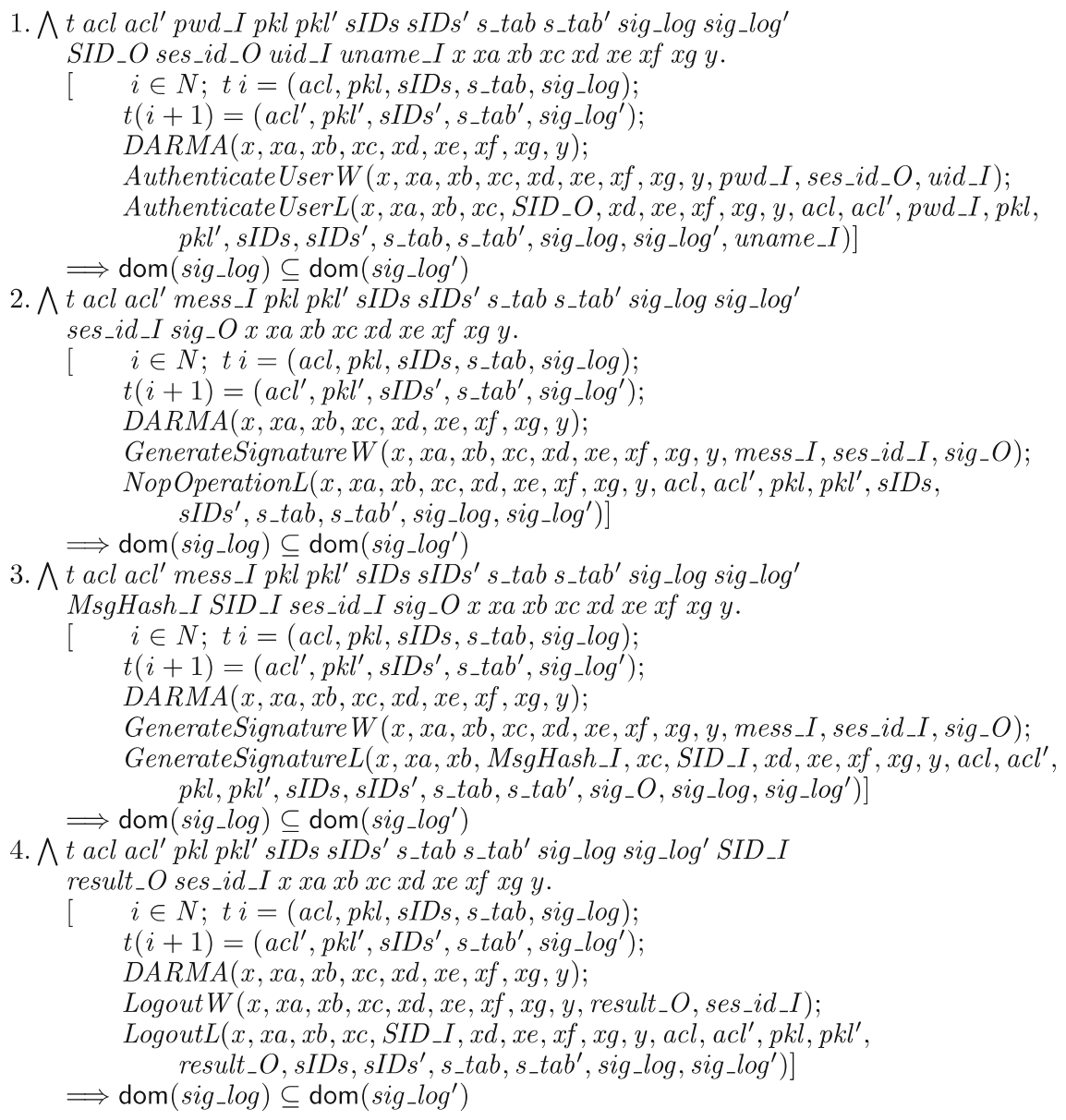

Fig. 15. Proof state after architectural decomposition

Specifically, this tactic matches the assumption

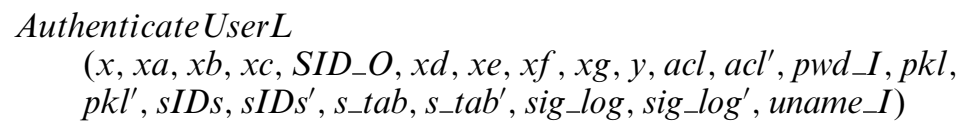

against the premise of AuthenticateUserL_inv_state_components. Afterwards it replaces this assumption by the instantiated conclusion of the lemma (performing renamings and adjusting bindings, as needed). Finally, the tactic performs simplifications, which closes this subgoal as sig_log $=$ sig_log $^{\prime}$ is now given as an assumption.

Our next two steps are similar and handle the second and fourth cases. ${ }^{10}$

by (zdtac NopOperationL_inv_state_components 1);

by(zdtac LogoutL_inv_state_components 2);

These apply analogous lemmas to show that the state component signature_log is not changed by a no-op operation or a logout on the server side.

The interesting step corresponds to the third subgoal, where the server generates a signature. Here we apply the following lemma (called GenerateSignatureL_siglog_mono), which specializes the postcondition of GenerateSignatureL: The operation may extend, but otherwise not alter, the signature log.

$\vdash$ GenerateSignatureL $\Rightarrow \operatorname{dom}($ signature_log $) \subseteq \operatorname{dom}($ signature_log' $)$

\footnotetext{
10 Note that the numbers associated with the subgoals are decremented each time a lower-numbered subgoal is proven. Hence, 1 here refers to the second subgoal and (since it is applied after two subgoals have been discharged) 2 refers to the fourth.
} 
Applying this lemma

by(zdtac GenerateSignatureL_siglog_mono 1);

closes the proof state. The following Isabelle operation extracts our claim as a theorem from the proof state and binds it to the name signature_log_mono, concluding our proof.

qed "signature_log_mono";

This example constitutes an instance of our general strategy for proving global invariants, presented in Sect. 3.5. Here, induction was not needed as it suffices to reason about an arbitrary pair of states without recourse to an induction hypothesis about previous pairs. The example shows how decomposition then suffices to reduce the main theorem proving problem to establishing local invariants of the individual schemas, i.e., conventional reasoning about preconditions and postconditions.

\section{References}

[AAG95] Abowd GD, Allen R, Garlan D (1995) Formalizing style to understand descriptions of software architecture. ACM Trans Softw Eng Methodol (TOSEM) 4(4):319-364

[ABL86] Abrial J-R, Börger E, Langmaack H (1986) Formal methods for industrial applications: Specifying and programming the steam boiler control, volume 1165 of Lecture Notes in Computer Science. Springer, Berlin Heidelberg New York

[Abr96] Abrial J-R (1996) The B-book: assigning programs to meanings. Cambridge University Press, Cambridge

[ACD $\left.{ }^{+} 99\right]$ Avrunin GS, Corbett JC, Dwyer MB, Pasareanu CS, Siegel SF (1999) Comparing finite-state verification techniques for concurrent software. Technical report, Amherst, MA, USA

[AH94] Alur R, Henzinger TA (1994) A really temporal logic. J ACM 41(1):181-203

[ASS ${ }^{+}$99] Arai T, Sekiguchi T, Satoh M, Inoue T, Nakamura T, Iwao H (1999) DARMA: Using different OSs concurrently based on nano-kernel technology. In: Proceedings of 59th-Annual Convention of information processing society of Japan, vol 1, pages 139-140. Information Processing Society of Japan, 1999 (in Japanese)

[BDG $\left.{ }^{+} 4\right]$ Brat G, Drusinsky D, Giannakopoulou D, Goldberg A, Havelund K, Lowry M, Pasareanu C, Venet A, Visser W, Washington R (2004) Experimental evaluation of verification and validation tools on martian rover software. Formal Methods Syst Des, 25(2-3):167-198

[BF00] Basin D, Friedrich S (2000) Combining WS1S and HOL. In Gabbay DM de Rijke M (eds) Frontiers of Combining Systems 2, vol 7 of Studies in Logic and Computation. Res Stud Press/Wiley, Baldock, Herts, UK, 39-56

[BH95] Bowen JP, Hinchey MG, (1995) Seven more myths of formal methods. IEEE Softw, 12(3):34-41

[BK91] Basin D, Kaufmann M, (1990) The Boyer-Moore Prover and Nuprl: an experimental comparison. In: Huet G, Plotkin G, (eds) Logical Frameworks. Cambridge University Press, Cambridge, pp. 90-119

[BKTW04] Basin D, Kuruma H, Takaragi K, Wolff B (2004) Specifying and verifying hysteresis signature system with HOL-Z. Technical Report 471, ETH Zürich. Available at the URL http://kisogawa.inf.ethz.ch/WebBIB/publications/papers/2005/HSD.pdf.

[BM88] Boyer RS, Moore JS (1988) Integrating decision procedures into heuristic theorem provers: a case study with linear arithmetic. Mach Intell (11):83-124

[BMV05] Basin D, Mödersheim S, Viganò L (2005) OFMC: A symbolic model checker for security protocols. International Journal of Information Security, 4(3):181-208 (Published online December 2004)

[BPW03] Backes M, Pfitzmann B, Waidner M (2003) A composable cryptographic library with nested operations. In CCS ’03: Proceedings of the 10th ACM conference on computer and communications security ACM Press, New York 220-230

[BRW03] Brucker AD, Rittinger F, Wolff B (2003) HOL-Z 2.0: A proof environment for Z-specifications. J Univ Comput Sci 9(2): 152-172

[BW03] Brucker AD, Wolff B (2003) A case study of a formalized security architecture. In: Electronic Notes in Theoretical Computer Science, vol 80. Elsevier, Amsterdam

[CAB ${ }^{+}$98] Chan W, Anderson RJ, Beame P, Burns S, Modugno F, Notkin D, Reese JD (1998) Model checking large software specifications. IEEE Trans Softw Eng 24(7):498-520

[Can01] Canetti R (2001) Universally composable security: a new paradigm for cryptographic protocols. In: FOCS '01: proceedings of the 42nd IEEE symposium on foundations of computer science. IEEE Computer Society p. 136

[Cor96] Corbett JC (1996) Evaluating deadlock detection methods for concurrent software. IEEE Trans Softw Eng 22(3):161-180

[CS05] Castellini C, Smaill A (2005) Proof planning for first-order temporal logic. In: Conference on automated deduction, vol 3632 of Lecture Notes in Computer Science, Springer, Berlin Heidelberg New York 235-249

[DAC99] Dwyer MB, Avrunin GS, Corbett JC (1999) Patterns in property specifications for finite-state verification. In: ICSE '99: Proceedings of the 21st international conference on software engineering. IEEE Computer Society Press, pp. 411-420

[Fis97] Fischer C (1997) CSP-OZ: A combination of Object-Z and CSP. In Proceedings of FMOODS'97: formal methods for open object-based distributed systems, vol 2, pages 423-438. Chapman \& Hall

[FORS] Fillitre J-C, Owre S,Rue H, Shankar N (2001) Ics: Integrated canonizer and solver. CAV 2001, number 2102 in Lecture Notes in Computer Science

[GO01] Gastin P, Oddoux D (2001) Fast LTL to Büchi automata translation. In: Berry G, Comon H, Finkel A (eds) Proceedings of the 13th conference on computer aided verification (CAV’01), number 2102 in Lecture Notes in Computer Science. Springer, Berlin Heidelberg New York pp. 53-65.

[Gup92] Gupta A (1992) Formal hardware verification methods: A survey. J Formal Methods Syst Des 1:151-238

[Hal90] Hall A (1990) Seven myths of formal methods. IEEE Softw 7(5):11-19

[Hol04] Holzmann GJ (2004) The SPIN model checker: primer and reference manual. Addison-Wesley, Boston 
[Int] International Standard ISO/IEC 13568:2002. Information technology — Z formal specification notation — syntax, type system and semantics.

[JS00] Jackson D, Sullivan K (2000) COM revisited: tool-assisted modelling of an architectural framework. In: ACM SIGSOFT Symposium on foundations of software engineering ACM Press, New York 149-158

[Low98] Lowe G (1998) Towards a completeness result for model checking of security protocols. In: PCSFW: Proceedings of the 11th computer security foundations workshop, IEEE Computer Society Press pp. 96-105

[MN95] Müller O, Nipkow T (1995) Combining model checking and deduction for I/O-automata. In Brinksma et al Ed (eds) Tools and algorithms for the construction and analysis of systems, 1st international workshop, TACAS'95, vol 1019 of Lecture Notes in Computer Science, Springer, Berlin Heidelberg New York, pp 1-16

[MP91] Manna Z, Pnueli A (1991) Completing the temporal picture. Theore Comput Sci J 83(1):97-130

[MP92] Manna Z Pnueli A (1992) The temporal logic of reactive and concurrent systems:specification. Springer, Berlin Heidelberg New York

[Nor] Norrish M (2003) Complete integer decision procedures as derived rules in HOL. In: Proceedings of TPHOLs'03, number 2758 in Lecture Notes in Computer Science, 71-86.

[NPW02] Nipkow T, Paulson LC, Wenzel M (2002) Isabelle/HOL - A proof assistant for higher-order logic, vol 2283 of Lecture Notes in Computer Science. Springer, Berlin Heidelberg New York

[ORR ${ }^{+}$96] Owre S, Rajan S, Rushby JM, Shankar N, Srivas MK (1996) PVS: Combining specification, proof checking, and model checking. In: Alur R, Henzinger TA (eds) Computer-aided verification, CAV '96, number 1102 in Lecture Notes in Computer Science, Berlin Heidelberg New York, pp 411-414

[Pau98] Paulson LC (1998) The inductive approach to verifying cryptographic protocols. J Comput Sec, 6:85-128

[Pe196] Peled D (1996) Combining partial order reductions with on-the-fly model checking. Formal Methods Sys Des 8:39-64

[Pro05] Promela proofs scripts for signature system case study. URL http://people.inf .ethz.ch/basin/spin-models .tar, 2005.

[RB99] Roscoe AW, Broadfoot PJ (1999) Proving security protocols with model checkers by data independence techniques. J Comput Sec 7(1):147-190

$\left[\mathrm{RSG}^{+}\right.$00] Ryan PYA, Schneider S, Goldsmith M, Lowe G, Roscoe AW (2000) The modelling and analysis of security protocols: the CSP approach. Addison-Wesley Reading

[RSS95] Rajan S, Shankar N, Srivas MK (1995) An integration of model-checking with automated proof checking. In: Wolper (ed), Computer-aided verification, CAV '95, vol 939 of Lecture Notes in Computer Science, Springer, Berlin Heidelberg New York, pp 84-97

[SBB ${ }^{+}$06] Sprenger C, Backes M, Basin D, Pfitzmann B, Waidner M (2006) Cryptographically sound theorem proving. In: 19th IEEE computer security foundations workshop, Venice, Italy IEEE Computer Society pp 153-166

[SD97] Smith G (1997) Derrick J (1997) Refinement and verification of concurrent systems specified in Object-Z and CSP. In: Proceedings of the international conference of formal engineering methods, IEEE Computer Society Press

[SG96] Shaw M, Garlan D (1996) Software architecture: perspectives on an emerging discipline. Prentice Hall, Englewood Cliffs

[SJO ${ }^{+}$05] Seger C-JH, Jones RB, O'Leary JW, Melham T, Aagaard MD, Barrett C, Syme D (2005) An industrially effective environment for formal hardware verification. IEEE Trans Comput Aided Des Integrated Circuits Syst, 24(9):1381-1405

[SM02] Susaki S, Matsumoto T (2002) Alibi establishment for electronic signatures. Inf Process Soc Jpn 43(8):2381-2393

[Spi92] Spivey JM (1992) The Z Notation: a reference manual. 2nd edn. Prentice-Hall International, New Jersey

[VW86] Vardi MY Wolper P (1986) Automata-theoretic techniques for modal logics of programs. J Comput Syst Sci 32:183-221

[WD96] Woodcock J, Davies J (1996) Using Z. Prentice-Hall International, New Jersey

[WVF97] Wing J, Vaziri-Farahani M (1997) A case study in model checking software systems. Sci Comput Program 28:273-299

Received 16 November 2005

Revised 03 August 2006

Accepted 19 September 2006 by M. J. Butler

Published online 13 January 2007 